COMMUNICATIONS IN

ANALYSIS AND GEOMETRY

Volume 10, Number 3, 475-514, 2002

\title{
Heat kernels and Green's functions on limit spaces
}

\author{
YU DING
}

In this paper, we study the behavior of the Laplacian on a sequence of manifolds $\left\{M_{i}^{n}\right\}$ with a lower bound in Ricci curvature that converges to a metric-measure space $M_{\infty}$. We prove that the heat kernels and Green's functions on $M_{i}^{n}$ will converge to some integral kernels on $M_{\infty}$ which can be interpreted, in different cases, as the heat kernel and Green's function on $M_{\infty}$. We also study the Laplacian on noncollapsed metric cones; these provide a unified treatment of the asymptotic behavior of heat kernels and Green's functions on noncompact manifolds with nonnegative Ricci curvature and Euclidean volume growth. In particular, we get a unified proof of the asymptotic formulae of Colding-Minicozzi, $\mathrm{Li}$ and $\mathrm{Li}$ Tam-Wang.

\section{Introduction.}

Assume $M^{n}$ is an $n$ dimensional Riemannian manifold with a lower bound in Ricci curvature,

$$
\operatorname{Ric}_{M^{n}} \geq-(n-1) \Lambda
$$

where $\Lambda \geq 0$. By the Bishop-Gromov inequality, we have a uniform volume doubling condition,

$$
\operatorname{Vol}\left(B_{2 R}(p)\right) \leq 2^{\kappa} \operatorname{Vol}\left(B_{R}(p)\right)
$$

here we can take $\kappa=n$ if $\Lambda=0$; if $\Lambda>0$, we require that $R$ is bounded from above, say, $R<D$ for some $D>0$. Moreover, there is a uniform Poincare inequality

$$
\frac{1}{\operatorname{Vol}\left(B_{R}(p)\right)} \int_{B_{R}(p)}\left|f-f_{p, R}\right| \leq \tau R\left(\frac{1}{\operatorname{Vol}\left(B_{R}(p)\right)} \int_{B_{R}(p)}|d f|^{2}\right)^{\frac{1}{2}}
$$

where $f_{p, R}$ is the average of $f$ on $B_{R}(p)$; see [Bu], [Ch3], [HaKo] and the references therein.

We assume, throughout this paper, $\left\{M_{i}^{n}\right\}$ is a sequence of complete Riemannian manifolds with (0.1) that converges in the pointed measured 
Gromov-Hausdorff sense, to a metric space $M_{\infty}$; we write $M_{i}^{n} \stackrel{d_{G H}}{\longrightarrow} M_{\infty}$, $d_{G H}$ is the Gromov-Hausdorff distance. In particular, (0.2) holds on $M_{\infty}$. One can show that (0.3) and the segment inequality, which is stronger than (0.3), hold on $M_{\infty}$ as well; note on $M_{\infty}$, the role of $|d u|$ in (0.3) is played by $g_{f}$, the minimal generalized upper gradient, see [Ch3], [ChCo4].

In Cheeger's paper [Ch3], a significant portion of analysis on smooth manifolds was extended to metric-measure spaces satisfying (0.2), (0.3). In [Ch3], [ChCo4] Cheeger and Colding defined a self-adjoint Laplacian operator $\Delta$ on $M_{\infty}$. By convention $\Delta$ is positive. They proved that the eigenvalues and eigenfunctions of the Laplacian $\Delta_{i}$ over $M_{i}^{n}$ converge to those on $M_{\infty}$, thereby establishing Fukaya's conjecture [Fu]. So if we consider $\mathcal{R I C}$, the completion under measured Gromov-Hausdorff convergence of the set of smooth manifolds with $(0.1)$, it is natural to expect that quantities associated to $\Delta$ should behave continuously.

In this paper, we will study this phenomenon in detail. Our main goal is to prove, in various cases, that the heat kernel $H_{i}$ and Green's function $G_{i}$ on $M_{i}^{n}$ converge uniformly to the heat kernel $H_{\infty}$ and Green's function $G_{\infty}$ on $M_{\infty}$. We will make precise the definition of these convergences in Section 1. For results concerning heat kernels, a lower bound in Ricci curvature (0.1) is enough; for Green's functions, we require that $\operatorname{Ric}_{M_{i}^{n}} \geq 0$; compare with (1.16), (1.18), (1.19).

Moreover, in the noncompact case, we also study the asymptotics of the heat kernel and Green's function on a manifold $M^{n}$ with $\operatorname{Ric}_{M^{n}} \geq 0$ and a Euclidean volume growth condition:

$$
\operatorname{Vol}\left(B_{R}(p)\right)>v_{0} R^{n}
$$

According to [ChCo1], any tangent cone at infinity of a manifold $M^{n}$ with $\operatorname{Ric}_{M^{n}} \geq 0$ and (0.4) is a metric cone $C(X)$. So viewed from a sufficiently large scale, $M^{n}$ appears to be close to some $C(X)$. Combined with the appropriate convergence theorems mentioned above, at a sufficiently large scale the heat kernel and Green's function on $M^{n}$ are close to those on $C(X)$.

On the other hand, we show that the classical analysis on cones, [Ch1], [Ch2], [ChTa1], can be generalized to $C(X)$. In particular, we have explicit expression of heat kernels and Green's functions on $C(X)$; see (6.21), (4.23). In this way we get a unified treatment for the asymptotic formulae of these integral kernels on $M^{n}$. In particular, we get new proofs of the ColdingMinicozzi asymptotic formula for Green's functions, [CoMi1] (compare with [LiTW]), the asymptotic formulae for heat kernels of Li [Li1] and Li-TamWang [LiTW]. 
The organization of this paper is as follows:

Section 1 reviews some background material that we need in the sequel.

In Section 2, in the compact case we prove, $H_{i}(\cdot, \cdot, t) \rightarrow H_{\infty}(\cdot, \cdot, t)$ uniformly (assuming (0.1)), and $G_{i} \rightarrow G_{\infty}$ uniformly, off the diagonal.(assuming $\operatorname{Ric}_{M^{n}} \geq 0$ ). It's well known that there is an eigenfunction expansion for heat kernels, so our results follows easily from the work of Cheeger-Colding [ChCo4], [Ch3], by estimating the remainders of the eigenvalue expansions. We remark, previously in [KK1], [KK2] it was proved that a subsequence of $H_{i}$ converges to some kernel on the compact metric space $M_{\infty}$.

By the Dirichlet's principle and the transplantation theorem of Cheeger [Ch3], we show in Section 3 that the uniform limit of solutions for Poisson equations is a solution for a Poisson equation, see also [Ch3], [ChCo4]. In particular, if $\left\{M_{i}^{n}\right\}$ are noncompact, $\operatorname{Ric}_{M_{i}^{n}} \geq 0$, satisfy (0.4) uniformly, then $G_{i} \rightarrow G_{\infty}$ uniformly off the diagonal (Theorem 3.21).

We treat the heat kernels on noncompact spaces in Section 5 . We assume (0.1). First, some subsequence of the Dirichlet heat kernels $H_{R, i}$ on $B_{R}\left(p_{i}\right) \subset M_{i}^{n}$ will converge to some function $H_{R, \infty}$ on $B_{R}\left(p_{\infty}\right) \subset M_{\infty}$. However, at present it is not clear if $H_{R, i}$ will converge. On the other hand by a generalized maximum principle, any two $H_{R, \infty}$ (from two different subsequences) can not be too different from each other, see (5.46). Letting $R \rightarrow \infty$, we prove that $H_{i}(\cdot, \cdot, t) \rightarrow H_{\infty}(\cdot, \cdot, t)$ in $L^{1}$.

In Theorem 5.59, when the noncollapsed limit $M_{\infty}$ is a manifold, we prove $H_{\infty}$ is the heat kernel over $M_{\infty}$, i.e. the integral kernel of the semigroup $e^{-t \Delta}$. For general $M_{\infty}$, the picture is not yet clear; however, it is true when $M_{\infty}=C(X)$ is a noncollapsed tangent cone that is the limit of a sequence of manifolds with nonnegative Ricci curvature, see Theorem 6.1.

In Section 4 and Section 6 we study the Laplacian on $C(X)$. We prove that in this case, one can still separate variables. We use these to study the structure of $G_{\infty}$ and $H_{\infty}$ on $C(X)$, and the asymptotic behavior of Green's function and heat kernel on a manifold $M^{n}$ with $\operatorname{Ric}_{M^{n}} \geq 0$ and (0.4).

In Section 7 we study the asymptotic behavior of the eigenvalues $\lambda_{j, \infty}$ on a compact metric space $M_{\infty}$ which is the limit of a sequence of manifolds $\left\{M_{i}^{n}\right\}$ with (0.1). We will prove in the noncollapsed case, the Weyl asymptotic formula is true on $M_{\infty}$; see Theorem 7.3. In the collapsed case, we get some link between the behavior of eigenvalues and $\operatorname{dim}_{M i n k}\left(M_{\infty}\right)$, the Minkowski dimension of $M_{\infty}$.

All of the estimates in this paper are uniform, i.e. the constants are valid for the whole family of manifolds we are considering (for example all compact manifolds $M^{n}$ with $\operatorname{Ric}_{M^{n}} \geq-(n-1) \Lambda$ and Diam $\left.M^{n} \leq D\right)$. 
Acknowledgments. I'm most grateful to my thesis advisor, Prof. Jeff Cheeger, for his encouragement and many valuable suggestions in connection with this work. Thanks also to Fengbo Hang for helpful conversations. I wish to thank the referees for pointing out the papers [LiTW] related to Theorem 6.30, and [KK1], [KK2] related to the compact case.

\section{Background and notation.}

Suppose $\left(M_{i}^{n}, \operatorname{Vol}_{i}\right) \stackrel{d_{G H}}{\longrightarrow}\left(M_{\infty}, \mu_{\infty}\right)$ in the measured Gromov-Hausdorff sense, i.e. the sequence $\left\{M_{i}^{n}\right\}$ converges in the Gromov-Hausdorff sense to $M_{\infty}$, and for any $x_{i} \rightarrow x_{\infty},\left(x_{i} \in M_{i}^{n}\right)$ and $R>0$, we have $\operatorname{Vol}_{i}\left(B_{R}\left(x_{i}\right)\right) \rightarrow$ $\mu_{\infty}\left(B_{R}\left(x_{\infty}\right)\right)$; here $\mu_{\infty}$ is Borel regular. In fact, for any sequences of manifolds with Ricci curvature bounded from below, after possible renormalization of the measures when $\left\{M_{i}^{n}\right\}$ is collapsing, one can alway find a subsequence converges in the measured Gromov-Hausdorff sense, see [ChCo2]. In the following we usually let Vol denote the (renormalized if $\left\{M_{i}^{n}\right\}$ is collapsing) measure on $M_{i}^{n}, i=1,2, \ldots, \infty$; on $M_{\infty}$ sometimes we also write $\mu_{\infty}$ for $\mathrm{Vol}_{\infty}$. We refer to [Ch3], [ChCo2], [Gr] for general background on measured Gromov-Hausdorff convergence.

Definition 1.1. Suppose $K_{i} \subset M_{i}^{n} \stackrel{d_{G H}}{\longrightarrow} K_{\infty} \subset M_{\infty}$ in the measured Gromov-Hausdorff sense. $f_{i}$ is a function on $M_{i}^{n}, i=1,2, \ldots ; f_{\infty}$ is a continuous function on $M_{\infty}$. Assume $\Phi_{i}: K_{\infty} \rightarrow K_{i}$ are $\epsilon_{i}$-Gromov-Hausdorff approximations, $\epsilon_{i} \rightarrow 0$. If $f_{i} \circ \Phi_{i}$ converge to $f_{\infty}$ uniformly, we say that $f_{i} \rightarrow f_{\infty}$ uniformly over $K_{i} \stackrel{d_{G H}}{\longrightarrow} K_{\infty}$.

For simplicity, in the above context, we also say that $f_{i} \rightarrow f_{\infty}$ uniformly on $K$; when we write $f_{i}(x) \rightarrow f_{\infty}(x)$, we mean that $f_{i} \rightarrow f_{\infty}$ uniformly and $f_{i}\left(x_{i}\right) \rightarrow f_{\infty}\left(x_{\infty}\right)$, where $x_{i} \rightarrow x_{\infty}, x_{i} \in M_{i}$.

In many applications, the family $\left\{f_{i}\right\}$ is actually equicontinuous. We remark, the Arzela-Ascoli theorem can be generalized to the case where the functions live on different spaces: when $M_{i}^{n} \stackrel{d_{G H}}{\longrightarrow} M_{\infty}$, for any bounded, equicontinuous sequence $\left\{f_{i}\right\}$ ( $f_{i}$ is a function on $M_{i}^{n}$ ), there is a subsequence that converges uniformly to some continuous function $f_{\infty}$ on $M_{\infty}$. The proof is straightforward.

We also introduce the notion of $L^{p}$ convergence $(1 \leq p \leq \infty)$.

Definition 1.2. We say $f_{i} \rightarrow f_{\infty}$ in $L^{p}$, if for all $\epsilon>0$, one can write $f_{i}=\phi_{i}+\eta_{i}$ such that $\phi_{i} \rightarrow \phi_{\infty}$ uniformly and $\lim \sup _{i \rightarrow \infty}\left\|\eta_{i}\right\|_{L^{p}} \leq \epsilon$, $\left\|\eta_{\infty}\right\|_{L^{p}} \leq \epsilon$. 
The following is a generalization of the Rellich-Kondrakov theorem:

Lemma 1.3 (Rellich). Assume $B_{1}\left(p_{i}\right) \subset M_{i}^{n} \stackrel{d_{G H}}{\longrightarrow} B_{1}(p) \subset M_{\infty}$ in the measured Gromov-Hausdorff sense. $u_{i}$ is a function on $M_{i}^{n}, i=1,2, \ldots$. Assume

$$
\int_{B_{1}\left(p_{i}\right)}\left(u_{i}\right)^{2}+\left|\nabla u_{i}\right|^{2} \leq N
$$

Then there is a subsequence of $\left\{u_{i}\right\}$ that converges in $L^{2}$ over any compact subset of the open balls $B_{1}$.

The proof depends only on a weak Poincare inequality (use a bigger ball on the right of $(0.3))$ and $(0.2)$. One can divide the ball $B_{R}(p)(R<1)$ into small subsets and approximate $f$ by functions that are constant over each of these small subsets, then one easily finds a convergent subsequence by standard diagonal arguments. Compare [Ch3], especially [CoMi3].

We use subscript $i$ and write $f_{i}, H_{R, i}, p_{i}$, etc. to denote functions, points, etc on $M_{i}^{n}$. To simplify notation, when we write an equation with some function or other objects with no subscription (for example, $f$ ), it should be understood that the equation is valid for some suitable convergent sequence of functions or other objects (for example, $\left\{f_{i}\right\} ; f_{i}$ is defined on $M_{i}^{n}, i=$ $1,2, \ldots)$, according to the context.

In [Ch3], Cheeger defined a Sobolev space $H_{1,2}$ on metric-measure spaces $(Z, \mu)$ satisfying $(0.2),(0.3)$, and proved that Lipschitz functions are dense in $H_{1,2}$. Denote by $\stackrel{\circ}{H}_{1,2}(\Omega)$ the closure in $H_{1,2}$ of Lipschitz functions supported in an open set $\Omega$. Recall in [Ch3], one has a natural finite dimensional cotangent bundle $T^{*} Z$. We use $d u$ to denote the differential of $u$, see Section 4 of [Ch3]. One can put a norm $|\cdot|$ on $T^{*} Z$ by assigning $|d f|=g_{f}=\operatorname{Lip} f$ for $f$ Lipschitz. Here as in [Ch3],

$$
\operatorname{Lip} f(x)=\limsup _{y \rightarrow x} \frac{|f(y)-f(x)|}{d(y, x)} .
$$

Note we use $\operatorname{Lip} f$ to denote the Lipschitz constant of $f$. Clearly, on smooth manifold $|\cdot|$ agrees with the standard norm $|d u|=|\nabla u|$. It was proved in [Ch3] that $|\cdot|$ is equivalent to a uniformly convex norm, in particular, an inner product.

If we have the stronger assumption $Z=M_{\infty}$ with $M_{i}^{n} \stackrel{d_{G H}}{\longrightarrow} M_{\infty}$, in [ChCo4] Cheeger and Colding proved that $M_{\infty}$ is $\mu_{\infty}$-rectifiable, and, as a 
corollary, the norm $|\cdot|$ actually comes from an inner product $\langle\cdot, \cdot\rangle$. So $H_{1,2}$ is made into a Hilbert space:

$$
\langle u, v\rangle_{H_{1,2}}=\int_{M_{\infty}} u v+\int_{M_{\infty}}\langle d u, d v\rangle,
$$

and for Lipschitz functions $f$, one has

$$
\|f\|_{H_{1,2}}^{2}=\|f\|_{L^{2}}^{2}+\int_{M_{\infty}}|\operatorname{Lip} f|^{2} .
$$

Now by the standard theory of Dirichlet forms, one gets a positive selfadjoint Laplacian $\Delta$ on $M_{\infty}$, see [Ch3], [ChCo4] for the details of this theory.

Recall one form of the transplantation theorem of Cheeger (for a proof, see Lemma 10.7 of [Ch3]):

Lemma 1.8. Assume $M_{i}^{n} \stackrel{d_{G H}}{\longrightarrow} M_{\infty} . \quad f_{\infty}$ is a Lipschitz function on $B_{R}\left(x_{\infty}\right) \subset M_{\infty}, x_{i} \rightarrow x_{\infty}$. Then there is a sequence of Lipschitz functions $\left\{f_{i}\right\}$ that converges uniformly to $f_{\infty}$, here $f_{i}$ is defined on $B_{R}\left(x_{i}\right) \subset M_{i}^{n}$. Moreover, one can require that

$$
\begin{gathered}
\limsup _{i \rightarrow \infty} \operatorname{Lip} f_{i} \leq \operatorname{Lip} f_{\infty}, \\
\limsup _{i \rightarrow \infty}\left\|\operatorname{Lip} f_{i}\right\|_{L^{2}} \leq\left\|\operatorname{Lip} f_{\infty}\right\|_{L^{2}} .
\end{gathered}
$$

By $[\mathrm{J}],[\mathrm{HaKo}]$, on length spaces satisfying $(0.2)$, a weak Poincare inequality implies a uniform Poincare-Sobolev inequality (i.e, put $L^{\chi p}$ norm on the left side of $(0.3)$ for some $\chi>1)$. In particular, we have a DirichletSobolev inequality for $u \in \stackrel{\circ}{H}_{1,2}\left(B_{R}(p)\right)$. So we have

Lemma 1.11 (Moser iteration). If for all $\phi$ with compact support in $B_{2}(p)$,

$$
\int \nabla u \nabla \phi=\int c u \phi+f \phi
$$

then for $q>C(\kappa)$, we have,

$$
\|u\|_{L^{\infty}\left(B_{1}(p)\right)} \leq C(n, q)(1+|c|)^{N(\tau, \kappa)}\left(\|u\|_{L^{2}\left(B_{2}(p)\right)}+\|f\|_{L^{q}\left(B_{2}(p)\right)}\right) .
$$


For proof, see chapter 4 of [Lin]. Note here we need to renormalize the measure.

Recall, on smooth manifolds we have

Lemma 1.14 (Gradient estimate). If $\Delta u-c u=f$, $\|u\|_{L^{2}}<\infty$ on $B_{2 R}(p)$ and $f$ is a $C^{2}$ function with Lipschitz constant $\operatorname{Lip} f, c$ is a constant, then on $B_{R}(p)$ we have a gradient estimate:

$$
|\nabla u| \leq C\left(\|f\|_{L^{\infty}},\|u\|_{L^{2}}, \operatorname{Lip} f\right)(1+|c|)^{N(\tau, \kappa)} .
$$

The proof follows a standard argument of Cheng-Yau, see [CY1], [LiY1], compare also with [Lin], [Li2], [SY]. Then use the Moser iteration to replace $\|u\|_{L^{\infty}}$ with $\|u\|_{L^{2}}$.

Finally recall the Li-Yau estimates [LiY2], [SY]: If $M^{n}$ satisfies (0.1), then its heat kernel $H$ satisfies

$$
H(x, y, t) \leq C(n) \operatorname{Vol}\left(B_{\sqrt{t}}(x)\right)^{-1 / 2} \operatorname{Vol}\left(B_{\sqrt{t}}(y)\right)^{-1 / 2} e^{-d^{2}(x, y) / 5 t} e^{C \Lambda t}
$$

If $t<T$, by volume comparison (1.16) simplifies to

$$
H(x, y, t) \leq C(n, \Lambda, T) \operatorname{Vol}\left(B_{\sqrt{t}}(x)\right)^{-1} e^{-d^{2}(x, y) / 5 t} e^{C \Lambda t} t^{-C(n)} e^{C(n, \Lambda, T) d(x, y)} .
$$

If we assume $\operatorname{Ric}_{M^{n}} \geq 0$, then

$$
\frac{C^{-1}(n)}{\operatorname{Vol}\left(B_{\sqrt{t}}(x)\right)} e^{-d^{2}(x, y) / 3 t} \leq H(x, y, t) \leq \frac{C(n)}{\operatorname{Vol}\left(B_{\sqrt{t}}(x)\right)} e^{-d^{2}(x, y) / 5 t}
$$

Assume $M^{n}$ is noncompact, write $G$ for the minimal positive Green's function on $M^{n}$. If $n \geq 3, M^{n}$ satisfies (0.4) and $\operatorname{Ric}_{M^{n}} \geq 0$, then $G$ exists ([LiY2], [LiT1], [LiT2], [SY]) and satisfies

$$
C^{-1}\left(v_{0}\right) d(x, y)^{2-n} \leq G(x, y) \leq C\left(v_{0}\right) d(x, y)^{2-n} .
$$

For proofs of the above estimates, see [LiY2], [SY].

\section{The compact case.}

In this section, we assume $M_{\infty}$ is compact. First we study the heat kernel $H$. 
It's well known that if $\operatorname{Ric}_{M_{i}^{n}} \geq-(n-1) \Lambda$, there is a lower bound for the $k$ th eigenvalue $\lambda_{k, i}$ of the Laplacian $\Delta_{i}$ over $M_{i}^{n}$ :

$$
\lambda_{k, i} \geq C(n, \Lambda, D) k^{\frac{2}{n}}
$$

here Diam $M_{i}^{n} \leq D$. The proof uses only (0.2) and (0.3); see [Gr] and Theorem 4.8 of [Ch3]. Hence over $M_{i}^{n}$ we have

$$
H_{i}(x, y, t)=\sum_{j=0}^{\infty} e^{-\lambda_{j, i} t} \phi_{j, i}(x) \phi_{j, i}(y),
$$

here $\phi_{j, i}$ is the eigenfunction of the $j$ th eigenvalue $\lambda_{j, i}$. By the CheegerColding spectral convergence theorem [ChCo4], for each $j, \lambda_{j, i} \rightarrow \lambda_{j, \infty}$, and $\phi_{j, i} \rightarrow \phi_{j, \infty}$ uniformly when $i \rightarrow \infty$, here $\lambda_{j, \infty}$ and $\phi_{j, \infty}$ are the $j$-th eigenvalue and (renormalized) eigenfunction of $\Delta$ on $M_{\infty}$. So (2.1) is also true for $\lambda_{j, \infty}$. Moreover,

$$
\begin{gathered}
\left\|\phi_{j, i}\right\|_{L^{\infty}} \leq C_{1}(n, \Lambda, D)\left(1+\lambda_{j, i}\right)^{C(n)}\left\|\phi_{j, i}\right\|_{L^{2}}, \\
\left\|\nabla \phi_{j, i}\right\|_{L^{\infty}} \leq C_{0}(n, \Lambda, D)\left(1+\lambda_{j, i}\right)^{C(n)}\left\|\phi_{j, i}\right\|_{L^{\infty}} .
\end{gathered}
$$

These are implied by (the proof of) the Moser iteration and the gradient estimate, see [LiY1]. By [ChCo4], (2.3), (2.4) can pass to $M_{\infty}$ (on $M_{\infty}(2.4)$ becomes an estimate for $\left.\operatorname{Lip} \phi_{j, \infty}\right)$. So it makes sense to write

$$
H_{\infty}(x, y, t)=\sum_{j=0}^{\infty} e^{-\lambda_{j, \infty} t} \phi_{j, \infty}(x) \phi_{j, \infty}(y) .
$$

By (2.1), $H_{\infty}$ is the heat kernel over $M_{\infty}$.

Apply (2.3), (2.4) to $\sum_{j=k}^{\infty} e^{-\lambda_{j, i} t} \phi_{j, i}(x) \phi_{j, i}(y)$, the tail of (2.2), one easily get

Theorem 2.6. Assume $M_{i}^{n} \stackrel{d_{G H}}{\longrightarrow} M_{\infty}$, $\operatorname{Ric}_{M_{i}^{n}} \geq-(n-1) \Lambda$. When $t>$ 0 fixed, $H_{i}$ converges to the heat kernel $H_{\infty}$ over $M_{\infty}$ uniformly. $H_{\infty}$ is continuous in $t, x, y$; when $t$ fixed, it is Lipschitz in $x, y$.

Corollary 2.7. For $H_{\infty}$ on $M_{\infty}$, the Li-Yau estimate (1.18) is true if $\operatorname{Ric}_{M_{i}^{n}} \geq 0$; if $\operatorname{Ric}_{M_{i}^{n}} \geq-(n-1) \Lambda$ then (1.16) is true. 
Next we study the Green's functions. Assume $\operatorname{Ric}_{M_{i}^{n}} \geq 0$. Recall,

$$
G_{i}(x, y)=\int_{0}^{\infty} h_{i}(x, y, t) d t
$$

here $h_{i}(x, y, t)=H_{i}(x, y, t)-\phi_{0, i}(x) \phi_{0, i}(y)=H_{i}(x, y, t)-1$. Note, since the sequence $\left\{M_{i}^{n}\right\}$ might collapse, we have to renormalize the measures and eigenvalues such that $\operatorname{Vol}\left(M_{i}^{n}\right)=1$ and $\left\{\phi_{j, i}\right\}_{j}$ is orthonormal, e.g. $\lambda_{0, i}=0$, $\phi_{0, i}=1$. So

$$
G_{i}(x, y)=\int_{0}^{\epsilon} h_{i}(x, y, t) d t+\sum_{j=1}^{\infty} e^{-\epsilon \lambda_{j, i} / 2} \int_{\frac{\epsilon}{2}}^{\infty} e^{-\lambda_{j, i} t} \phi_{j, i}(x) \phi_{j, i}(y) d t
$$

When $x$ is fixed, by (2.3),

$$
\sum_{j=k}^{\infty}\left\|e^{-\epsilon \lambda_{j, i} / 2} \int_{\frac{\epsilon}{2}}^{\infty} e^{-\lambda_{j, i} t} \phi_{j, i}(x) \phi_{j, i}(y) d t\right\|_{L^{\infty}} \leq \sum_{j=k}^{\infty} \frac{e^{-\epsilon \lambda_{j, i}}}{\lambda_{j, i}} C_{1}\left(1+\lambda_{j, i}\right)^{C_{3}} .
$$

This goes to 0 uniformly in $i$ as $k \rightarrow \infty$, by (2.1). On the other hand, clearly (1.18) holds after renormalization; when Ric $\geq 0, R<1 / 8$ we have the (rescaled) volume bound

$$
C(n, D) R^{n} \leq \operatorname{Vol}\left(B_{R}(x)\right) \leq \sqrt{R} C(n) \operatorname{Vol}\left(B_{\sqrt{R}}(x)\right) \leq C^{\prime}(n) R .
$$

So when $d(x, y) \geq \delta>0$, by (1.18),

$$
\int_{0}^{\epsilon}\left|h_{i}(x, y, t)\right| d t \leq \epsilon+\int_{0}^{\epsilon}\left|H_{i}(x, y, t)\right| d t \leq \epsilon+C \int_{0}^{\epsilon} t^{-\frac{n}{2}} e^{-\frac{\delta^{2}}{5 t}} d t
$$

So in particular, by choosing $\epsilon$ small, we get a function $G_{\infty}(x, y)$ on $M_{\infty}$, such that $G_{i} \rightarrow G_{\infty}$ in $L^{\infty}$ on compact subsets, off the diagonal.

Finally, we want to check $G_{\infty}$ is the Green's function over $M_{\infty}$. We now establish an $L^{1}$ bound for $G(x, y)$ over the ball $B_{R}(x)$. Note

$$
\int_{B_{R}(x)}|G(x, y)| d y \leq \int_{B_{R}(x)} \int_{0}^{1}|h(x, y, t)| d t d y+\int_{B_{R}(x)} \int_{1}^{\infty}|h(x, y, t)| d t d y .
$$


Since $\|\phi(y)\|_{L^{2}}=1$, by (2.1), (2.3), (2.11) and the Schwartz inequality,

$$
\begin{aligned}
& \int_{B_{R}(x)} \int_{1}^{\infty}|h(x, y, t)| d t d y \\
& \leq \sum_{j=1}^{\infty} \int_{B_{R}(x)} e^{-\lambda_{j} / 2}\left|\phi_{j}(x)\right| \int_{\frac{1}{2}}^{\infty} e^{-\lambda_{j} t}\left|\phi_{j}(y)\right| d t d y \\
& \leq C(n) \sum_{j=1}^{\infty} e^{-\lambda_{j} / 2}\left(1+\lambda_{j}\right)^{C_{2}(n)} \sqrt{R} \int_{\frac{1}{2}}^{\infty} e^{-\lambda_{j} t} d t \leq C^{\prime}(n) \sqrt{R} .
\end{aligned}
$$

Now we focus on the first term on the right hand side of (2.13). Since $H-h=1$, and we have (2.11), it's enough to estimate the integral of $H$. Put $R<1 / 8$, by (1.18), (2.11) we have

$$
\begin{aligned}
& \int_{0}^{1} \int_{B_{R}(x)} H(x, y, t) d t d y \\
& \leq \int_{0}^{1} \int_{B_{R}(x)} C(n) \mathrm{Vol}^{-1}\left(B_{\sqrt{t}}(x)\right) e^{-\frac{d(x, y)^{2}}{5 t}} d t d y \\
& \leq\left(\int_{0}^{R} \int_{B_{R}(x)} C(n) \mathrm{Vol}^{-1}\left(B_{\sqrt{t}}(x)\right) e^{-\frac{d(x, y)^{2}}{5 t}} d t d y\right)+C^{\prime}(n) \sqrt{R} .
\end{aligned}
$$

Next,

$$
\int_{0}^{R} \frac{\int_{B_{R}(x)} e^{-d(x, y)^{2} / 5 t} d y}{\operatorname{Vol}\left(B_{\sqrt{t}}(x)\right)} d t=\int_{0}^{R} \frac{\int_{0}^{R} e^{-r^{2} / 5 t} A(r) r^{n-1} d r}{\int_{0}^{\sqrt{t}} A(r) r^{n-1} d r} d t
$$

here $A(r) r^{n-1}$ is the surface area element of $\partial B_{r}(x)$. Since $\operatorname{Ric}_{M_{i}^{n}} \geq 0, A(r)$ is non-increasing. The right hand side of (2.16) can be bounded by

$$
\begin{aligned}
& \int_{0}^{R}\left(\sum_{s=0}^{[R / \sqrt{t}]+1} \int_{s \sqrt{t}}^{(s+1) \sqrt{t}} e^{-r^{2} / 5 t} A(r) r^{n-1} d r / \int_{0}^{\sqrt{t}} A(r) r^{n-1} d r\right) d t \\
& \leq C_{1}(n) R+\int_{0}^{R} \sum_{s=1}^{[R / \sqrt{t}]+1} e^{-s^{2} / 5}\left((s+1)^{n}-s^{n}\right) d t \leq C^{\prime}(n) R .
\end{aligned}
$$

So combine (2.14), (2.15) and (2.11) we get

$$
\int_{B_{R}(x)}|G(x, y)| d y \leq C^{\prime}(n) \sqrt{R} .
$$


Since $G_{i} \rightarrow G_{\infty}$ uniformly off the diagonal, use the Cheeger-Colding theorem on the convergence of eigenfunctions [ChCo4] and (2.3), (2.4), we get, for all $x$,

$$
\begin{aligned}
\phi_{j, \infty}(x)=\lim _{i \rightarrow \infty} \phi_{j, i}(x) & =\lim _{i \rightarrow \infty} \int_{M_{i}} G_{i}(x, y) \lambda_{j, i} \phi_{j, i}(y) d y \\
& =\int_{M_{\infty}} G_{\infty}(x, y) \lambda_{j, \infty} \phi_{j, \infty}(y) d y .
\end{aligned}
$$

So $G_{\infty}$ is the Green's function over $M_{\infty}$. Moreover, by (2.10), (2.12) and Lemma 1.14, $G_{\infty}$ is Lipschitz continuous off the diagonal. It is harmonic off the diagonal by Lemma 3.17. So we have proved

Theorem 2.20. Assume $M_{i}^{n} \stackrel{d_{G H}}{\longrightarrow} M_{\infty}$, Ric $M_{i}^{n} \geq 0$. Then the Green's function $G_{\infty}$ on $M_{\infty}$ exists. On any compact subsets $K$ off the diagonal, $G_{\infty}$ is Lipschitz and harmonic, $G_{i} \rightarrow G_{\infty}$ uniformly on $K$.

\section{The Green's functions on noncompact spaces.}

Recall how on a manifold, one solves the the Poisson equation,

$$
\Delta u_{R}=f,\left.u_{R}\right|_{\partial B_{R}(p)}=h,
$$

for Lipschitz functions $f, h$ on the closed ball $B_{R}(p)$. By the Dirichlet's principle, $u_{R}$ is the unique minimizer of the functional

$$
I(u)=\int_{B_{R}(p)}\left(|d u|^{2}-f u\right) .
$$

within the space $\mathcal{E}=h+\stackrel{\circ}{H}_{1,2}\left(B_{R}(p)\right)$, note $\Delta$ is positive by convention.

Assume $M_{i}^{n} \stackrel{d_{G H}}{\longrightarrow} M_{\infty}$ in the measured Gromov-Hausdorff sense, $\operatorname{Ric}_{M_{i}^{n}} \geq$ $-(n-1) \Lambda$. Recall, by [Ch3], [ChCo4], $\Delta$ is linear on $M_{\infty}$. So the above variational method is valid also on $M_{\infty}$.

Lemma 3.3 (Lower semicontinuity of energy). Suppose $u_{i}, f_{i}$ are $C^{2}$ functions over $M_{i}^{n}, \Delta u_{i}=f_{i}, u_{i} \rightarrow u_{\infty}, f_{i} \rightarrow f_{\infty}$ uniformly over the sequence of converging balls $B_{2 R}\left(p_{i}\right) \rightarrow B_{2 R}\left(p_{\infty}\right)$, and there is a uniform gradient estimate for $u_{i}$ and $f_{i}$ :

$$
\left|\nabla u_{i}\right|,\left|\nabla f_{i}\right|<L
$$


Then we have

$$
I\left(u_{\infty}\right) \leq \liminf _{i \rightarrow \infty} I\left(u_{i}\right)
$$

Proof. As in [ChCo1], we can get an integral bound for the Hessian of $f_{i}$ on the ball $B_{1}\left(p_{i}\right)$ : recall the Bochner formula

$$
\frac{1}{2} \Delta\left(\left|\nabla f_{i}\right|^{2}\right)=\left|\operatorname{Hess}_{f_{i}}\right|^{2}+<\nabla \Delta f_{i}, \nabla f_{i}>+\operatorname{Ric}\left(\nabla f_{i}, \nabla f_{i}\right) .
$$

Multiply by a cut-off function $\phi$ with $\operatorname{supp} \phi \subset B_{r} \subset B_{1}\left(q_{i}\right),\left.\phi\right|_{B_{r / 2}}=1$, $|\nabla \phi| \leq c(n, r),|\Delta \phi| \leq c(n, r)$; see Theorem 6.33 of [ChCo1]. Since $f_{i}$ is harmonic,

$$
\frac{1}{2} \phi \Delta\left(\left|\nabla f_{i}\right|^{2}\right)=\phi\left|\operatorname{Hess}_{f_{i}}\right|^{2}+\phi \operatorname{Ric}\left(\nabla f_{i}, \nabla f_{i}\right) .
$$

Integrate by parts,

$$
\int_{B_{r}} \frac{1}{2}\left(\left|\nabla f_{i}\right|^{2}\right) \Delta \phi=\int_{B_{r}} \phi\left|\operatorname{Hess}_{f_{i}}\right|^{2}+\int_{B_{r}} \phi \operatorname{Ric}\left(\nabla f_{i}, \nabla f_{i}\right) .
$$

By assumption, there is a definite lower bound for the last term in (3.8). Note $|\Delta \phi|$ is uniformly bounded by construction, we have a uniform upper bound for $\int_{B_{r}} \phi\left|\operatorname{Hess}_{f_{i}}\right|^{2}$. So by Lemma 1.3 we can assume some subsequence of $\left|\nabla f_{i}\right|$ converges to a function $\Gamma$ on $B_{R}\left(p_{\infty}\right) \subset M_{\infty}$ in $L^{2}$. Assume, $x \in \mathcal{R}_{k} \subset M_{\infty}$ for some $k$ (all tangent cone at $x$ is $\mathbf{R}^{k}$ ), there is some subset $A(x) \subset M_{\infty}$ such that and $\Gamma$ is continuous on $A(x), x \in A(x)$ is a density point of $A(x)$. By Luzin's theorem and the results in [ChCo2], these properties hold for almost all $x \in M_{\infty}$. For such $x$, we prove

$$
\left|\operatorname{Lip} f_{\infty}(x)\right| \leq \Gamma(x) .
$$

Clearly, (3.9) implies our lemma.

To prove (3.9), it's enough to prove, for all $\psi>0$, if $l=d(x, y)$ is sufficiently small, then

$$
\left|f_{\infty}(x)-f_{\infty}(y)\right| \leq d(y, x)(\Gamma(x)+6 \psi) .
$$

By the gradient estimate of $f_{i}$ (so of $f_{\infty}$ ), if (3.10) is not true for some $y_{0}$, then for all $y \in B_{l \psi / L}\left(y_{0}\right)$,

$$
\left|f_{\infty}(x)-f_{\infty}(y)\right|>d(y, x)(\Gamma(x)+5 \psi) .
$$


Pick $x_{i}, y_{0, i} \in M_{i}^{n}, x_{i} \rightarrow x, y_{0, i} \rightarrow y_{0}, d\left(x_{i}, y_{0, i}\right)=l$. Then for $i$ big enough, for all $y_{i} \in B_{l \psi / L}\left(y_{0, i}\right)$ and all minimal geodesic $\gamma_{i}$ connecting $x_{i}$ and $y_{i}$,

$$
\int_{\gamma_{i}}\left|\nabla f_{i}\right| \geq d\left(x_{i}, y_{i}\right)(\Gamma(x)+4 \psi)
$$

First of all, since $\left|\nabla f_{i}\right|$ is uniformly bounded by $L$, a simple computation shows along every $\gamma_{i}$ we must have

$$
\left|\nabla f_{i}\right|>\Gamma(x)+2 \psi
$$

on a subset of $\gamma_{i}$ which has 1-Hausdorff measure at least $2 \psi l /(L-\Gamma(x))$. Put

(3.14) $T_{i}=\left\{v \in T_{x_{i}} \mid v=\gamma^{\prime}(0)\right.$ for some minimal geodesic $\gamma$

$$
\text { from } \left.x_{i} \text { to } y_{i} \in B_{l \psi / L}\left(y_{0, i}\right)\right\} \text {. }
$$

We must have

$$
H^{n-1}\left(T_{i}\right)>C(n, L, \psi) H^{n-1}\left(\partial B_{1}(0)\right),
$$

where $H^{n-1}$ is the $(n-1)$-Hausdorff measure over the unit sphere $\partial B_{1}(0)$ in the tangent space $T_{x_{i}}$. Combine this with (3.13), when $M_{\infty}$ is noncollapsed, if $l$ is small enough, by the proof of the Bishop-Gromov inequality, for sufficiently big $i$,

$$
\frac{\operatorname{Vol}\left(\left\{z_{i} \in B_{l}\left(x_{i}\right)|| \nabla f_{i}\left(z_{i}\right) \mid>\Gamma(x)+2 \psi\right\}\right)}{\operatorname{Vol}\left(B_{l}\left(x_{i}\right)\right)} \geq C(x, n, L, \psi)>0 .
$$

Now $\left|\nabla f_{i}\right|$ converge to $\Gamma$ in $L^{2}$, so (3.16) is also true if we substitute $\left|\nabla f_{i}\right|$ in (3.16) by $\Gamma, x_{i}$ by $x$. We get a contradiction to the choice of $x$.

The proof is the same when $M_{\infty}$ is collapsed. We just use the segment inequality ([ChCo1], [ChCo4]) to get (3.16) from (3.11), (3.12) and (3.13).

Lemma 3.17. Let $u_{\infty}, f_{\infty}$ be as in the previous lemma. Then

$$
\Delta u_{\infty}=f_{\infty}
$$


Proof. Assume this is not true over a ball $B_{\lambda}\left(p^{*}\right) \subset \subset B_{1}(0)$. By solving the Dirichlet problem on $B_{\lambda}\left(p^{*}\right)$ we can find $v_{\infty}$ with the same boundary value as $u_{\infty}$ over $\partial B_{\lambda}$, but with smaller energy, say

$$
I\left(v_{\infty}\right)=\int_{B_{\lambda}\left(p^{*}\right)}\left|d v_{\infty}\right|^{2}-f_{\infty} v_{\infty}<\int_{B_{\lambda}\left(p^{*}\right)}\left|d u_{\infty}\right|^{2}-f_{\infty} u_{\infty}-2 \Psi
$$

By obvious density properties, we can change $v_{\infty}$ slightly so that $v_{\infty}$ agrees with $u_{\infty}$ on a neighborhood of $\partial B_{\lambda}\left(p^{*}\right)$. By Lemma 3.3, for $i$ big enough,

$$
I\left(v_{\infty}\right) \leq I\left(u_{i}\right)-\Psi .
$$

So by (the proof of) Lemma 1.8 (see Section 10 of [Ch3]), for $i$ big enough we can find a function $v_{i}$ with the same boundary value on $\partial B_{i}$ as $u_{i}$ but with smaller energy $I$. That contradicts the fact that $\Delta u_{i}=f_{i}$.

The solution of (3.1) is unique on $M_{\infty}$ because the maximum principle holds, see Section 7 of [Ch3].

We now study the Green's functions. Assume $\left(M_{i}^{n}, p_{i}, \mathrm{Vol}_{i}\right) \stackrel{d_{G H}}{\longrightarrow}$ $\left(M_{\infty}, p, \mu_{\infty}\right)$ in the pointed measured Gromov-Hausdorff sense ([Gr], [ChCo2]), where $\operatorname{Ric}_{M_{i}^{n}} \geq 0, M_{i}^{n}$ is complete, noncompact, $n \geq 3$.

Theorem 3.21. Assume, $M_{i}^{n}$ also satisfies $\operatorname{Vol}\left(B_{R}\left(p_{i}\right)\right)>v_{0} R^{n}$. Then on $M_{\infty}$ there is a Green's function $G_{\infty}, G_{i} \rightarrow G_{\infty}$ uniformly on any compact subsets of $M \times M$ that does not intersects with the diagonal.

Proof. Since $n \geq 3$, the Euclidean volume growth condition (0.4) implies that the minimal positive Green's function $G_{i}$ exists on $M_{i}^{n}$ ( $\Delta$ is positive). Moreover, $G_{i}$ satisfies the Li-Yau estimate (1.19). So by the Cheng-Yau gradient estimate and the Arzela-Ascoli theorem, for any fixed $x$, for some subsequence (still denoted by $G_{i}$ ), we have

$$
G_{i}(x, y) \rightarrow G_{\infty}(x, y)
$$

uniformly over any compact set in $M \backslash\{x\}$. Clearly, $G_{\infty}$ satisfies (1.19). We will show $G_{\infty}$ is in fact well defined, and $G_{i} \rightarrow G_{\infty}$ as stated in the above theorem.

Assume $f_{\infty}$ is any Lipschitz function supported in $B_{K}\left(p_{\infty}\right) \subset M_{\infty}$, $\operatorname{Lip} f_{\infty} \leq L$. By Lemma 1.8 and approximation, there is a sequence of $C^{2}$ functions $\left\{f_{i}\right\}$ with $f_{i} \rightarrow f_{\infty}$ uniformly, $\operatorname{Lip} f_{i} \leq 2 L, \operatorname{supp} f_{i} \subset B_{2 K}\left(p_{i}\right) \subset$ $M_{i}^{n}, i=1,2, \ldots, \infty$. 
Recall on each manifold $M_{i}^{n}$ with maximal volume growth condition, the function,

$$
u_{i}(x)=\int_{M_{i}^{n}} G_{i}(x, y) f_{i}(y) d y,
$$

solves the Poisson equation

$$
\Delta u_{i}=f_{i}, \lim _{x \rightarrow \infty} u_{i}(x)=0 .
$$

Now by the Li-Yau estimate (1.19) and the Euclidean volume growth condition (0.4), $G_{i}$ is locally integrable, so $u_{i}$ is uniformly bounded. The gradient estimate Lemma 1.14 shows that $u_{i}$ are uniformly Lipschitz:

$$
\operatorname{Lip} u_{i} \leq C(L, K, n) \text {. }
$$

So we can find a subsequence of $\left\{u_{i}\right\}$ that converges to some Lipschitz function $u_{\infty}$ on $M_{\infty}$. Note that by the Li-Yau estimate, (1.19),

$$
\left|u_{i}(x)\right| \leq C^{\prime}(L, K, n) d\left(x, p_{i}\right)^{2-n}, \quad(i=1,2, \ldots, \infty) .
$$

So by Lemma 3.17, $\Delta u_{\infty}=f_{\infty}$ on $M_{\infty}$. Using the fact Laplacian is linear, by (3.26) and the maximum principle (Section 7 of [Ch3]), it is clear that $u_{\infty}$ is well defined and $u_{i} \rightarrow u_{\infty}$ uniformly.

Notice, by (1.19),

$$
u_{\infty}(x)=\int_{M_{\infty}} G_{\infty}(x, y) f_{\infty}(y) d y .
$$

Since we can choose arbitrary $K, f_{\infty}$, clearly $G_{\infty}$ is also well defined, $G_{i} \rightarrow G_{\infty}$ uniformly, off the diagonal. By (3.27) and Lemma 3.17, $G_{\infty}$ can be interpreted as the minimal positive Green's function on $M_{\infty}$.

\section{Separation of variables on tangent cones.}

Assume $M_{i}^{n}$ is complete noncompact, $\operatorname{Ric}_{M_{i}^{n}} \geq 0$ and satisfies (0.4) uniformly, $M_{i}^{n} \stackrel{d_{G H}}{\longrightarrow} M_{\infty}$. Recall that by [ChCo1], [ChCo2], every tangent cone of $M_{\infty}$ is a metric cone. We denote such a cone by $C(X)=\mathbf{R}_{+} \times_{r} X$, here $\left(X, d x^{2}\right)$ is a compact length space with $\operatorname{Diam} X \leq \pi$, [ChCo1]. The metric on $C(X)$ is

$$
d \rho^{2}=d r^{2}+r^{2} d x^{2}
$$


Here we write $r$ for the distance from the pole $p_{\infty}=(0, X)$.

The measure $\mu_{\infty}$ on $C(X)$ is just the $n$-Hausdorff measure, [ChCo2]. Since we can rescale $C(X), \mu_{\infty}$ induced a natural measure $\mu_{X}$ on $X$ that obviously satisfies a doubling condition (0.2) (with some different $\kappa$ ). Moreover, $X$ satisfies the rectifiability properties as stated in Section 5 of [ChCo4].

Also recall from [Ch3], for $f, g \in H_{1,2}$,

$$
d(f g)=f \cdot d g+g \cdot d f
$$

Moreover, from [ChCo4] and [Ch3], if $f$ is a function depending only on $r$ and $g$ is a function independent of $r$, then by the polar identity, one gets $\langle d f, d g\rangle=0$.

Lemma 4.3 (Weak Poincare inequality). For $B_{R} \subset X, 3 R<\frac{1}{5}, f \in$ $H_{1,2}(X)$,

$$
\int_{B_{R}(x)}\left|f-f_{x, R}\right|^{2} \leq \tau_{X} R^{2} \int_{B_{3 R(x)}}|d f|^{2}
$$

Proof. Define, for $x \in X$,

$$
\operatorname{Box}((1, x), a, b)=\left\{(t, y) \in C(X)|| t-1 \mid<a, d_{X}(x, y)<b\right\} .
$$

Put

$$
\mathrm{Box}_{1}=\operatorname{Box}((1, x), R, R), \operatorname{Box}_{2}=\operatorname{Box}((1, x), 3 R, 3 R) \subset C(X)
$$

So $\operatorname{Box}_{1} \subset B_{2 R}((1, x)) \subset \operatorname{Box}_{2}$. We extend $f$ to be a $H_{1,2}$ function independent of $r$ on $C(X)$. Assume $f_{\text {Ball }}$ is the average of $f$ on the ball $B_{2 R}((1, x)) \subset C(X)$,

$$
\begin{aligned}
\int_{B_{R}(x)} \mid & f-\left.f_{x, R}\right|^{2}=C(n) R^{-1} \int_{\mathrm{Box}_{1}}\left|f-f_{x, R}\right|^{2} \leq C(n) R^{-1} \int_{\mathrm{Box}_{1}}\left|f-f_{\mathrm{Ball}}\right|^{2} \\
& \leq C(n) R^{-1} \int_{B_{2 R}((1, x))}\left|f-f_{\mathrm{Ball}}\right|^{2} \leq C(n) \tau R \int_{B_{2 R}((1, x))}|d f|^{2} \\
& \leq C(n) \tau R \int_{\mathrm{Box}_{2}}|d f|^{2}=\tau_{X} R^{2} \int_{B_{3 R}(x)}|d f|^{2} .
\end{aligned}
$$

The first and last identity come from the Fubini theorem. Note $f_{x, R}$ is also the average of $f$ over Box $_{1}$, and we used the Poincare inequality on $C(X)$ in the middle inequality. 
We remark, a weak Poincare inequality is already enough for many purposes. Since $X$ is a length space, by [HaKo] one has (0.3) on $X$. As in [Ch3], [ChCo4], we define a positive operator $\Delta_{X}$ on $X$. Note by $(0.2),(0.3)$ the compact embedding lemma 1.3 is true on $X$. So by the standard elliptic theory, on $X$ we have a basis $\left\{\phi_{j}\right\}_{j=0}^{\infty}$ for $L^{2}(X)$ and a sequence $\mu_{j} \rightarrow \infty$ such that $\Delta_{X} \phi_{j}=\mu_{j} \phi_{j}$, compare [Ch3], [ChCo4]. Moreover, one can do Moser iteration on $X$, so $\phi_{i}$ is Hölder continuous; see [Lin], [GT]. These have applications in Section 6.

Next we show, even the cross section $X$ may not be a manifold, there is still a separation of variables formula for $\Delta$ on $C(X)$. See [Ch1] for the classical case.

Recall that $\langle\cdot, \cdot\rangle$ is the inner product on $T^{*} M_{\infty}$ as in [Ch3], [ChCo4].

\section{Lemma 4.8.}

$$
\Delta(f g)=f \Delta g+g \Delta f-2\langle d f, d g\rangle
$$

Proof. Since $d(f g)=f \cdot d g+g \cdot d f$, for any Lipschitz (or $H_{1,2}$ ) function $\phi$ with compact support, we have (recall $\Delta$ is positive)

$$
\int\langle d f, g \cdot d \phi+\phi \cdot d g\rangle-\int g \phi \Delta f=0 .
$$

Exchange the role of $f$ and $g$, we get

$$
\int\langle d(f g), d \phi\rangle-\int \phi(f \Delta g+g \Delta f-2\langle d f, d g\rangle)=0 .
$$

Similarly, by $d(f \circ g)=f^{\prime}(g) d g$, we get

$$
\Delta f \circ g=-f^{\prime \prime}(g)|d g|^{2}+f^{\prime}(g) \Delta g .
$$

Lemma 4.13. On $C(X), r^{2-n}$ is harmonic away from the pole.

Proof. By the results in Section 4 of [ChCo1], $r^{2-n}$ is the uniform limit of a sequence of harmonic functions $\mathcal{G}$. So by the proof of Lemma $3.17, r^{2-n}$ is harmonic.

By the maximum principle on $X$ (Section 7 in [Ch3]), we have 
Lemma 4.14. If $X$ is compact, and $\Delta_{X} f=0$, then $f$ is a constant.

Theorem 4.15. Assume $u$ lies in the ring generated by functions of the form $u=f g$ where $f$ depends only on $r$ and $g$ depends only on $x$. Then on $C(X) \backslash\left\{p_{\infty}\right\}$,

$$
\Delta u=-\frac{\partial^{2} u}{\partial r^{2}}-\frac{n-1}{r} \frac{\partial u}{\partial r}+\frac{1}{r^{2}} \Delta_{X} u
$$

Proof. Compare [ChTa1]. By (4.12) and Lemma 4.13, on the cone $C(X)$ we have

$$
\Delta f(r)=-f^{\prime \prime}(r)-f^{\prime} \frac{n-1}{r} .
$$

Next we apply Lemma 4.8 , recall $\langle d f, d g\rangle=0$. We pick a test function $\phi$ of the form $\phi=a(r) b(x)$. By scaling we see, $\Delta g(R, x)=R^{-2} \Delta g(1, x)$. Assume $a$ is supported over the interval $[\alpha, \beta]$,

$$
\int_{C(X)}\langle d g, d \phi\rangle=\int_{\alpha}^{\beta}\left(t^{1-n} \int_{X} t^{-2}\langle d g, a(t) d b\rangle d x\right) d t
$$

here in the second integral we view $g$ and $b$ as functions on the cross section $X=(1, X)$. So we compute

$$
\int_{C(X)}\langle d g, d \phi\rangle=\int_{\alpha}^{\beta}\left(t^{-n-1} \int_{X} a(t) b(x) \Delta_{X} g d x\right) d t=\int \phi r^{-2} \Delta_{X} g
$$

Since we can choose arbitrary $a, b$, and $\Delta g(R, x)=R^{-2} \Delta g(1, x)$, we get

$$
\Delta g(R, x)=R^{-2} \Delta_{X} g .
$$

This suffices to complete the proof.

Using transformation $D_{R}:(r, x) \mapsto(R r, x)$, we deduce from the existence and uniqueness of $G_{\infty}$ that

$$
G_{\infty}\left(D_{R} x, D_{R} y\right)=R^{2-n} G_{\infty}(x, y) .
$$

So $G_{\infty}\left(p_{\infty}, x\right)=d\left(p_{\infty}, x\right)^{2-n} g(x)$ for some Lipschitz function $g$. By (4.16) and Lemmas $4.8,4.14, g=C$ is a constant. 


\section{Corollary 4.22.}

$$
G_{\infty}\left(p_{\infty}, x\right)=(n-2)^{-1} \mu_{X}(X)^{-1} d^{2-n}\left(p_{\infty}, x\right) .
$$

Proof. We know $G_{\infty}\left(p_{\infty}, x\right)=C d^{2-n}\left(p_{\infty}, x\right)$. We construct a test function $\phi=\phi(r)$ such that $\phi$ is a smooth function of $r, \phi=1$ for $r$ small and $\phi=0$ for $r \geq 1$. So

$$
\begin{aligned}
1 & =\int G\left(p_{\infty}, y\right) \Delta \phi(y)=\int_{0}^{1}\left(-\phi^{\prime \prime}-\frac{n-1}{r} \phi^{\prime}\right) C r^{2-n} r^{n-1} \mu_{X}(X) d r \\
& =-C \mu_{X}(X) \int_{0}^{1}(n-2) \phi^{\prime} d r=(n-2) C \mu_{X}(X) .
\end{aligned}
$$

Corollary 4.25. Assume $\Delta u=f$ on $B_{R}\left(p_{\infty}\right) \backslash\left\{p_{\infty}\right\}, f \in L^{\infty}$, and

$$
\lim _{x \rightarrow p}|u(x)| d\left(x, p_{\infty}\right)^{n-2}=0 .
$$

Then $\Delta u=f$ on $B_{R}\left(p_{\infty}\right)$.

Proof. By the De Giorgi-Nash-Moser theorem, $u$ is bounded and Hölder continuous. In our case, $G_{\infty}\left(p_{\infty}, x\right)=C d\left(p_{\infty}, x\right)^{2-n}$, so the proof goes exactly like the $\mathbf{R}^{n}$ case (where the maximum principle is used). For details see [Lin].

Relation (4.23) implies the Colding-Minicozzi asymptotic formula, [CoMi1], compare [LiTW]. In fact, we rescale the manifold $M^{n}$ to get a sequence of manifolds that converges to $C(X)$, a tangent cone at infinity, see [ChCo2]. By Theorem 3.21, the new (rescaled) Green's functions converge to the Green's function on $C(X)$.

Theorem 4.27 (Colding-Minicozzi). On a noncompact manifold $M^{n}$ with $\operatorname{Ric}_{M^{n}} \geq 0$ and (0.4) we have

$$
\lim _{d(x, p) \rightarrow \infty} d(x, p)^{n-2} G(p, x)=(n-2)^{-1}\left(n \lim _{R \rightarrow \infty} R^{-n} \operatorname{Vol}\left(B_{R}(p)\right)\right)^{-1}
$$

Note the tangent cones may not be unique; in collapsing case, a tangent cone might not be a metric cone, [ChCo2], [Per]. 


\section{Heat kernels on noncompact spaces.}

We assume in this section, all the manifolds $M^{n}$ are noncompact, satisfying (0.1). On $M^{n}$, write $H(x, y, t)$ for the heat kernel; we denote by $H_{R}(x, y, t)$ the Dirichlet heat kernel on the metric ball $B_{R}(p)$, put $H_{R}=0$ outside $B_{R}(p)$.

One technical issue is, the boundary $\partial B_{R}(p)=d^{-1}(R)$ may not be smooth, here $d=d(p, \cdot)$. However, we can approximate $d$ by a Morse function $d_{\epsilon}$, see [Hir], and (assuming) $R$ is not a critical value, etc. So in the sequel we always assume the boundary are smooth.

Lemma 5.1. Assume $\operatorname{Ric}_{M^{n}} \geq-(n-1) \Lambda$. Then there is a function $\epsilon(t, \Lambda, R)$ with $\lim _{R \rightarrow \infty} \epsilon(t, \Lambda, R)=0$ for $t>0$, and

$$
\int_{M-B_{R}(x)} H(x, y, t) d y \leq \epsilon(t, \Lambda, R)
$$

Proof. By the Bishop-Gromov inequality, it's easy to see

$$
\operatorname{Vol}\left(B_{\sqrt{t}}(x)\right) \leq C_{1}(n, \Lambda, t) e^{C_{2}(n, \Lambda, t) d(x, y)} \operatorname{Vol}\left(B_{\sqrt{t}}(y)\right) .
$$

Put $s_{\Lambda}(r)=(1 / \sqrt{\Lambda}) \sinh \sqrt{\Lambda} r$. We now use the Li-Yau estimate (1.16):

$$
\begin{aligned}
\int_{M-B_{R}(x)} H(x, y, t) d y \\
\leq C^{\prime}(n, \Lambda, t) \int_{M-B_{R}(x)} \operatorname{Vol}^{-1}\left(B_{\sqrt{t}}(x)\right) e^{-d(x, y)^{2} / 5 t} e^{C_{2}(n, \Lambda, t) d(x, y)} \\
=C^{\prime}(n, \Lambda, t) \int_{R}^{\infty} e^{-r^{2} / 5 t} e^{C_{2} r} A(r) s_{\Lambda}^{n-1}(r) d r / \int_{0}^{\sqrt{t}} A(r) s_{\Lambda}^{n-1}(r) d r \\
\leq C^{\prime} \int_{R}^{\infty} e^{-r^{2} / 5 t} e^{C_{2} r} s_{\Lambda}^{n-1}(r) d r / \int_{0}^{\sqrt{t}} s_{\Lambda}^{n-1}(r) d r=\epsilon(t, \Lambda, R) .
\end{aligned}
$$

Here $A(r) s_{\Lambda}^{n-1}(r)$ is the surface area element of $\partial B_{R}(x)$. We used the fact $A(r)$ is non-increasing (Bishop-Gromov inequality) and assumed, without lose of generality, $R>\sqrt{t}$.

Lemma 5.5. Let $\left(M^{n}, p\right)$ be a noncompact complete manifold. Then

$$
\lim _{R \rightarrow \infty} H_{R}(x, \cdot, t)=H(x, \cdot, t) .
$$


The convergence is uniform, and uniformly in $L^{1}$, on any finite interval $t \in[0, T]$.

Proof. Assume $R>\max \{T, 2 d(x, p)\}$. Put

$$
M(R)=\sup \left\{H(x, y, t) \mid y \in \partial B_{R}(x), 0<t \leq T\right\},
$$

by (1.17) and volume comparison we have

$$
M(R) \leq \sup _{0<t \leq T} C(n, \Lambda, T) t^{-C_{1}(n)} e^{-R^{2} / 5 t} e^{C_{2}(n, \Lambda, T) R} \operatorname{Vol}\left(B_{R}(p)\right)^{-1},
$$

so $\lim _{R \rightarrow \infty} M(R) \operatorname{Vol}\left(B_{R}(p)\right)=0$. By the maximum principle,

$$
H(x, y, t)-M(R) \leq H_{R}(x, y, t) \leq H(x, y, t) .
$$

Combining this with Lemma 5.1, we have

$$
\left\|H_{R}(x, \cdot, t)-H(x, \cdot, t)\right\|_{L^{1}}<\epsilon(n, \Lambda, T, R),
$$

and $\lim _{R \rightarrow \infty} \epsilon(n, \Lambda, T, R)=0$.

Assume $\lambda_{j}$ is the $j$-th Dirichlet eigenvalue of the Laplacian on $B_{R}(p), \phi_{j}$ is the corresponding eigenfunction, $\left\|\phi_{j}\right\|_{L^{2}\left(B_{R}(p)\right.}=1$.

Lemma 5.11. There exists a constant $C(n, \Lambda, R)$ such that

$$
C(n, \Lambda, R)^{-1} R^{-2} k^{\frac{2}{n}} \leq \lambda_{k} \leq C(n, \Lambda, R) R^{-2} k^{2} .
$$

Proof. Since $R$ fixed, we have $\operatorname{Vol}\left(B_{r}(x)\right) \geq C_{0}(n, \Lambda, R) r^{n} \operatorname{Vol}\left(B_{R}(p)\right)$, for $r<2 R$ and $B_{r}(x)$ with nonempty intersection with $B_{R}(p)$. Then since $H_{R} \leq H$, we can follow the heat kernel argument as in page 178 of [SY] to get the lower bound of $\lambda_{k}$.

The upper bound follows from an argument of Cheng, see page 105 of [SY].

Lemma 5.13. For any $N>0$, there is a function $\epsilon(N, \Lambda, R, \delta)$ such that for any fixed $R, \lim _{\delta \rightarrow 0} \epsilon(N, \Lambda, R, \delta)=0$, and for $k$ such that $\lambda_{k}<N$,

$$
\int_{A(p, R-\delta, R)}\left|\phi_{k}\right|^{2} \leq \epsilon(N, \Lambda, R, \delta) .
$$

Here $A(p, R-\delta, R)$ is the annulus $\{z \mid R-\delta \leq d(p, z) \leq R\}$. 
Proof. By (1.16) and the Bishop-Gromov inequality, when $t=1$,

$$
\begin{aligned}
\int_{\mathrm{A}(p, R-\delta, R)}\left|\phi_{k}\right|^{2} & \leq e^{\lambda_{k}} \int_{\mathrm{A}(p, R-\delta, R)} H(x, x, 1) d x \\
& \leq e^{N} \int_{\mathrm{A}(p, R-\delta, R)} \frac{C(n, \Lambda, R)}{\operatorname{Vol}\left(B_{R}(p)\right)} d x \leq \epsilon(N, \Lambda, R, \delta) .
\end{aligned}
$$

As before, assume $M_{i}^{n} \stackrel{d_{G H}}{\longrightarrow} M_{\infty}$ in the pointed measured GromovHausdorff sense, $M_{i}$ is noncompact, satisfies (0.1). Write $\lambda_{j, i}$ for the $j$-th Dirichlet eigenvalue over $B_{R}\left(p_{i}\right) \subset M_{i}^{n} . \phi_{j, i}$ is the corresponding eigenvalue:

$$
\Delta \phi_{j, i}=\lambda_{j, i} \phi_{j, i} ; \int_{B_{R}\left(p_{i}\right)} \phi_{j, i} \phi_{k, i}=\delta_{j k} .
$$

Lemma 5.17. For fixed $j, k>0$, assume (for a subsequence of the eigenvalues), $\lambda_{j, i} \rightarrow \lambda_{j, \infty}, \lambda_{k, i} \rightarrow \lambda_{k, \infty}$. Then there is a subsequence (denoted also by $\left.\phi_{j, i}, \phi_{k, i}\right)$ that converges uniformly on compact subsets of $B_{R}$, and also in $L^{2}\left(B_{R}\right)$, to two locally Lipschitz functions $\phi_{j, \infty}, \phi_{k, \infty}$. Moreover,

$$
\Delta \phi_{j, \infty}=\lambda_{j, \infty} \phi_{j, \infty}, \Delta \phi_{k, \infty}=\lambda_{k, \infty} \phi_{k, \infty}, \int_{B_{R}(p)} \phi_{j, \infty} \phi_{k, \infty}=\delta_{j k} .
$$

Proof. The results is clear in view of Lemma 5.11, Lemma 1.14 and Lemma 3.17. The $L^{2}$ convergence and the orthonormal property for the limit functions are implied by locally uniform convergence and Lemma 5.13 .

By Lemma 5.11, we can assume, after passing to a subsequence, that every eigenvalue and eigenfunction converge:

$$
\lim _{i \rightarrow \infty} \lambda_{j, i}=\lambda_{j, \infty}, \lim _{i \rightarrow \infty} \phi_{j, i}=\phi_{j, \infty}
$$

Write

$$
H_{R, \infty}=\sum_{j=1}^{\infty} e^{-\lambda_{j, \infty} t} \phi_{j, \infty}(x) \phi_{j, \infty}(y) .
$$

For all fixed $t, x$, by Lemma 5.11 and Lemma 1.11, Lemma 1.14,

$$
H_{R, i}(x, \cdot, t) \rightarrow H_{R, \infty}(x, \cdot, t) .
$$


The convergence is in $L^{2}$, and is locally uniform. Note we don't know if $H_{R, \infty}$ (and $\phi_{j, \infty}, \lambda_{j, \infty}$ ) is well defined. For the moment (before Lemma 5.40), we fix, by a diagonal argument, one sequence $R_{k} \rightarrow \infty$, and one subsequence $\left\{M_{i_{v}}^{n}\right\}$ of $\left\{M_{i}^{n}\right\}$ such that for each $k, H_{R_{k}, i} \rightarrow H_{R_{k}, \infty}$. For simplicity, we just write $\left\{M_{i}^{n}\right\}$ for this subsequence of manifolds. So by the results on smooth manifolds, for $R_{j}<R_{k}$,

$$
0 \leq H_{R_{j}, \infty}(x, y, t) \leq H_{R_{k}, \infty}(x, y, t) \leq \frac{C(n, \Lambda) e^{-d^{2}(x, y) / 5 t} e^{C \Lambda t}}{\operatorname{Vol}_{\infty}^{1 / 2}\left(B_{\sqrt{t}}(x)\right) \operatorname{Vol}_{\infty}^{1 / 2}\left(B_{\sqrt{t}}(y)\right)} .
$$

Thus we can also assume that the nondecreasing sequence $H_{R_{j}, \infty}$ converges pointwise to some function $H_{\infty}$. We will prove that $H_{\infty}$ is well defined.

By (5.9) and the locally uniform convergence of $H_{R, i}$ to $H_{R, \infty}(5.21)$, the $\mathrm{Li}$-Yau estimate (1.16) is also true for $H_{\infty}$ :

$$
0 \leq H_{\infty}(x, y, t) \leq \frac{C(n, \Lambda) e^{-d^{2}(x, y) / 5 t} e^{C \Lambda t}}{\operatorname{Vol}_{\infty}^{1 / 2}\left(B_{\sqrt{t}}(x)\right) \operatorname{Vol}_{\infty}^{1 / 2}\left(B_{\sqrt{t}}(y)\right)} .
$$

Note we need to renormalize the measures whenever $\left\{M_{i}^{n}\right\}$ is collapsing. Clearly, when $\operatorname{Ric}_{M_{i}^{n}} \geq 0$, we also have a lower bound of $H_{\infty}$ as in (1.18).

\section{Corollary 5.24.}

$$
\int_{M_{\infty}} H_{\infty}(x, z, s) H_{\infty}(z, y, t-s) d z=H_{\infty}(x, y, t) .
$$

Proof. By (5.21), (5.25) is true for $H_{R, \infty}$. Write $H_{\infty}(x, z, s)=$ $H_{R, \infty}(x, z, s)+\epsilon_{R}^{1}(z)$, similarly $H_{\infty}(z, y, t-s)=H_{R, \infty}(z, y, t-s)+\epsilon_{R}^{2}(z)$, here $H_{R, \infty}=0$ outside $B_{R}\left(p_{\infty}\right), \epsilon_{R}^{1}, \epsilon_{R}^{2} \geq 0$ are two functions. In view of Lemmas 5.1, 5.5, (5.21) and (5.23),

$$
\begin{gathered}
\limsup _{R \rightarrow \infty}\left(\left\|\epsilon_{R}^{1}(z)\right\|_{L^{1}}+\left\|\epsilon_{R}^{2}(z)\right\|_{L^{1}}\right)=0, \\
\left\|\epsilon_{R}^{1}(z)\right\|_{L^{\infty}}+\left\|\epsilon_{R}^{2}(z)\right\|_{L^{\infty}}<C\left(t, s, M_{\infty}\right) .
\end{gathered}
$$

Now (5.25) is clear.

\section{Corollary 5.27 .}

$$
\int_{M_{\infty}} H_{\infty}(x, y, t) d y=1
$$


Proof. By (5.21), Lemmas 5.1 and 5.5.

Lemma 5.29. For any Lipschitz function $f$ with compact support,

$$
\left|\int_{M_{\infty}} H_{R, \infty}(x, y, t) f(y) d y-f(x)\right| \leq \epsilon\left(t,\|f\|_{\left.L^{\infty}, \operatorname{Lip} f\right) .}\right.
$$

Here for any $F, L>0, \lim _{t \rightarrow 0} \epsilon(t, F, L)=0$. The conclusion is also true for $H_{\infty}$.

Proof. By an argument similar to those given in Lemma 5.1 and Lemma 5.5. Note on smooth manifolds, when $t \rightarrow 0$, the integral of $H_{R}$ is smaller than, but almost equal to 1 , and tends to concentrate on smaller and smaller balls centered at $x$. In view of (5.21) and the Li-Yau estimate (5.23), we easily get (5.30).

Let the Sobolev space $\stackrel{\delta}{H}_{1,2}\left(B_{R}\left(p_{\infty}\right)\right)$ be defined as in [Ch3], i.e., the $H_{1,2}$ closure of the set of Lipschitz functions supported in the interior of $B_{R}\left(p_{\infty}\right)$,

Lemma 5.31. The space $\stackrel{\circ}{H}_{1,2}\left(B_{R}\left(p_{\infty}\right)\right)$ is contained in $\Phi$, the $L^{2}$-linear span of functions $\phi_{j, \infty}$. In particular, any Lipschitz function with support in $B_{R-\delta}$ lies in $\Phi$.

Proof. If not, by approximation, we have a Lipschitz function $f_{\infty}$ with compact support and an $\epsilon>0$ such that

$$
\sum_{j=1}^{\infty}\left(\int_{B_{R}\left(p_{\infty}\right)} f_{\infty} \phi_{j, \infty}\right)^{2}<(1-3 \epsilon)\left\|f_{\infty}\right\|_{L^{2}}^{2} .
$$

Using Lemma 1.8, we can transplant $f_{\infty}$ back to a Lipschitz function, $f_{i}$, on $M_{i}^{n}$, with compact support which is close to $f_{\infty}$ in $L^{\infty}$, such that the energy of $f_{i}$ is close to that of $f_{\infty}$. Write

$$
f_{i}=\sum_{j=1}^{N} a_{j, i} \phi_{j, i}+R_{N, i}, \quad R_{N, i}=\sum_{j=N+1}^{\infty} a_{j, i} \phi_{j, i} .
$$

Notice,

$$
\lim _{i \rightarrow \infty} a_{j, i}=\int_{M_{\infty}} f_{\infty} \phi_{j, \infty}
$$


So by the min-max principle and Lemma $5.11, \lim _{i \rightarrow \infty}\left\|\nabla f_{i}\right\|_{L^{2}}=\infty$, we get a contradiction to the construction of $f_{i}$, Lemma 1.8.

Remark, it is not clear if we have $\phi_{j, \infty} \in \stackrel{\circ}{H}_{1,2}$.

Now for Lipschitz functions $f_{i}$ with compact support in $B_{R}\left(p_{i}\right) \subset M_{i}^{n}$ $(i=1,2, \ldots, \infty), f_{i} \rightarrow f_{\infty}$ uniformly, we have

$$
f_{i}=\sum_{j=1}^{\infty} a_{j, i} \phi_{j, i}, \quad a_{j, i}=\int_{M_{i}^{n}} f_{i} \phi_{j, i} .
$$

So $a_{j, i} \rightarrow a_{j, \infty}$. Clearly,

$$
\int_{B_{R}\left(p_{i}\right)} H_{R, i}(x, y, t) f_{i}(y) d y=\sum_{j=1}^{\infty} e^{-\lambda_{j, i} t} a_{j, i} \phi_{j, i}(x) .
$$

We say $h(x, t)$ is a locally strong solution, if $h$ continuous, Lipschitz in $x, \frac{\partial h}{\partial t}$ exists, continuous on $M \times \mathbf{R}^{+}$, and when $t$ fixed, $-\Delta h=\frac{\partial h}{\partial t}$, i.e.

$$
\int_{\Omega} \psi \frac{\partial h}{\partial t}+\int_{\Omega}\left\langle d_{x} h, d_{x} \psi\right\rangle=0
$$

for all Lipschitz functions $\psi$ with compact support.

By Lemma 5.11, Lemma 1.11 and 1.14,

$$
\lim _{k \rightarrow \infty} \sum_{j=k}\left|e^{-\lambda_{j} t} \phi_{j}(x) d_{y} \phi_{j}(y)\right|=0 .
$$

So $H_{R, i}$ is a locally strong solution of the heat equation. Similarly the function,

$$
h_{i}(x, t)=\int_{B_{R}} H_{R, i}(x, y, t) f_{i}(y) d y \quad(i=1,2, \ldots, \infty),
$$

is also a locally strong solution. Note for the case $i=\infty$ we used also Lemma 5.17 .

For locally strong solutions on $M_{\infty}$, there is also a weak maximum principle:

Lemma 5.40. Assume $h$ is a locally strong solution on $B_{2 R} \times[0, T+1]$, then if

$$
\left.h\right|_{B_{R} \times\{0\}} \leq 0,\left.h\right|_{\partial B_{R} \times[0, T]} \leq 0 .
$$

Then $h \leq 0$ on $B_{R} \times[0, T]$. 
Proof. Define

$$
m(s)=\sup \left\{h(x, s) \mid x \in B_{R}, \frac{\partial h}{\partial t}(x, s) \leq 0\right\} .
$$

Since $h, \frac{\partial h}{\partial t}(x, s)$ are continuous functions, it's easy to show that $m$ is nonincreasing and $m(0)=0$ implies $m(s) \leq 0$ for all $s>0$. Now by the weak maximum principle for Poisson equations (see [GT], [Ch3] or (5.37)), we have, when $s$ fixed,

$$
\sup \left\{h(x, s) \mid x \in B_{R}\right\}=m(s) \leq 0 .
$$

Now we can address the uniqueness of $H_{\infty}$. Recall that $\left(M_{i}, p_{i}\right) \rightarrow$ $\left(M^{\infty}, p_{\infty}\right)$. Assume for $R>0$, we got two limits $H_{4 R, \infty}^{(1)}, H_{4 R, \infty}^{(2)}$ through different subsequences of manifolds.

Theorem 5.44. For $x, y \in B_{R}\left(p_{\infty}\right), t<T$, there is an $\epsilon(R)>0$ such that

$$
\begin{gathered}
\lim _{R \rightarrow \infty} \epsilon(R)=0 \\
H_{4 R, \infty}^{(1)}(x, y, t)<H_{4 R, \infty}^{(2)}(x, y, t)+\epsilon(R) .
\end{gathered}
$$

Proof. We can assume $R>T^{2}>t^{2}$ and $R>4$. Assume (5.46) is not true, then there is a point $a \in B_{R}\left(p_{\infty}\right)$ and $0<r<1$ such that

$$
H_{4 R, \infty}^{(1)}(x, y, t) \geq H_{4 R, \infty}^{(2)}(x, y, t)+\epsilon(R),
$$

for $y \in B_{2 r}(a)$. We then construct a test function $f \geq 0$ such that, $f$ Lipschitz, supported in $B_{r}(a)$,

$$
2 \int_{B_{r}(a)} f \geq \operatorname{Vol}\left(B_{r}(a)\right) \sup _{B_{r}(a)} f .
$$

Clearly, for $R<\infty$, the functions,

$$
F_{k}(z, s)=\int_{B_{r}(a)} H_{4 R, \infty}^{(k)}(z, y, s) f(y) d y,(k=1,2),
$$


are locally strong solutions of the heat equation, and (by the construction of $f$ ),

$$
F_{1}(x, t) \geq F_{2}(x, t)+\epsilon \int_{B_{r}(a)} f \geq F_{2}(x, t)+\frac{\epsilon(R)}{2} \operatorname{Vol}\left(B_{r}(a)\right) \sup _{B_{r}(a)} f .
$$

For a point $z$ near $\partial B_{2 R}\left(p_{\infty}\right)$, say $d(z, p)=2 R, d(a, z) \geq R$,

$$
F_{k}(z, s)<\operatorname{Vol}\left(B_{r}(a)\right) \frac{C(n)}{\operatorname{Vol}\left(B_{\sqrt{s}}(z)\right)} e^{-R^{2} / 5 s} e^{C R} \sup _{B_{r}(a)} f,(k=1,2) .
$$

By a standard argument of the Bishop-Gromov inequality,

$$
F_{k}(z, s)<C_{1}(n) \frac{\operatorname{Vol}\left(B_{r}(a)\right)}{\operatorname{Vol}\left(B_{1}(p)\right)} s^{-\frac{n}{2}} e^{-R^{2} / 5 s} e^{C^{\prime} R} \sup _{B_{r}(a)} f,(k=1,2) .
$$

Next we consider the case that $s$ is small. Since $f$ is fixed, by (5.30), $F_{k} \rightarrow f$ uniformly on $B_{2 R}(p)$ when $s \rightarrow 0$.

In view of the weak maximum principle on $B_{2 R}\left(p_{\infty}\right) \times[0, T]$ (Lemma $5.40)$, clearly we should choose $\epsilon(R)$ such that that for $0 \leq s \leq T$,

$$
\frac{C_{1}(n)}{\operatorname{Vol}\left(B_{1}(p)\right)} s^{-\frac{n}{2}} e^{-R^{2} / 5 s} e^{C^{\prime} R}<\frac{\epsilon(R)}{4},
$$

by the maximum principle we got a contradiction to (5.50).

Theorem 5.54. $H_{\infty}$ is well defined. For fixed $t>0, x_{i} \rightarrow x_{\infty}$, we have $H_{i}\left(x_{i}, \cdot, t\right) \rightarrow H_{\infty}\left(x_{\infty}, \cdot, t\right)$ in $L^{1}$. When $H_{\infty}$ is continuous, this convergence is also uniform.

Proof. By the previous theorem and the construction of $H_{\infty}$ (compare (5.9)), we see $H_{\infty}$ is independent of the choice of subsequences, so well defined.

We already know, by (5.9), (5.21), (5.23), that locally $H_{i} \rightarrow H_{\infty}$ in $L^{1}$. The proof of global $L^{1}$ convergence is similar with Lemma 5.1, Lemma 5.5, using (1.18), (5.23).

Recall (see [SY] Chapter 4), there is a Harnack inequality

$$
H_{i}\left(x, y_{1}, t_{1}\right) \leq H_{i}\left(x, y_{2}, t_{2}\right)\left(\frac{t_{2}}{t_{1}}\right)^{n} \exp \left(\frac{d^{2}\left(y_{1}, y_{2}\right)}{4\left(t_{2}-t_{1}\right)}+C(n, \Lambda)\left(t_{2}-t_{1}\right)\right),
$$

for $0<t_{1}<t_{2}$. If $H_{\infty}$ is continuous, then locally $H_{\infty}$ is uniformly continuous (especially, with respect to $t$ ), clearly by (5.55) the convergence $H_{i} \rightarrow H_{\infty}$ must be uniform, compare with (5.23). 
We now want to interpret the meaning of $H_{\infty}$. Recall from [ChCo4] and [Ch3], $\Delta$ is a positive self-adjoint operator. So $-\Delta$ generates a semigroup $e^{-t \Delta}$.

Assume $f_{i}$ is supported in $B_{K}\left(p_{i}\right) \subset B_{R}\left(p_{i}\right)$. Use the notation in (5.35), define

$$
W_{R, i}(t) f_{i}(x)=\sum_{j=1}^{\infty} a_{j, i} \cos \left(\sqrt{\lambda_{j, i}} t\right) \phi_{j, i} .
$$

By the finite speed of propagation (see [Ta]), when $t$ is fixed and $R>K+t$, $W_{R, i}(t) f$ is independent of $R$. We write $W_{i}(t) f$ for $W_{R, i}(t) f$ with $R$ big. For $i<\infty$,

$$
e^{-t \Delta} f_{i}(x)=\int_{M_{i}^{n}} H_{i}(x, y, t) f_{i}(y) d y=\int_{-\infty}^{\infty} e^{-s^{2} / 4 t} W_{i}(s) f_{i}(x) d s
$$

see $[\mathrm{CGT}]$, [Ta]. Define

$$
W_{R, \infty}(t) f_{\infty}(x)=\sum_{j=1}^{\infty} a_{j, \infty} \cos \left(\sqrt{\lambda_{j, \infty}} t\right) \phi_{j, \infty} .
$$

We notice that $W_{R, i}(i=1,2, \ldots, \infty)$ does not increase $L^{2}$ norm, and we should use Lemma 1.8 and approximation to construct $C^{2}$ functions $f_{i}$ on $M_{i}^{n}$ that converges to $f_{\infty}$. Clearly, $W_{R, i} f_{i} \rightarrow W_{R, \infty} f_{\infty}$ in $L^{2}$. We remark that generally, we don't know if $W_{R, \infty}$ is well defined.

Theorem 5.59. If the limit $M_{\infty}$ is a smooth manifold, and the limit measure is the canonical measure on $M_{\infty}$, then $H_{\infty}$ is the heat kernel on $M_{\infty}$.

Proof. In the noncollapsing case, by Colding's theorem [Co], the limit measure is the canonical measure on $M_{\infty}$; when $M_{\infty}=\mathbf{R}^{k}$ for some $k$, the limit measure is also a multiple of the standard Lebesgue measure on $\mathbf{R}^{k}$, see [ChCo2]. In these cases, the Laplacian we defined on $M_{\infty}$ is the same one from the original smooth structure of $M_{\infty}$.

Pick any $C_{0}^{\infty}$ function $f$ supported in $B_{R}$, So

$$
\int_{M_{\infty}}\left(\Delta^{k}\right) f \phi_{j, \infty}=\left(\lambda_{j, \infty}\right)^{k} \int_{M_{\infty}} f \phi_{j, \infty}=\left(\lambda_{j, \infty}\right)^{k} a_{j, \infty} .
$$

Since $\left(\Delta^{k}\right) f \in C_{0}^{\infty}$, we have for all $k, \lim _{j \rightarrow \infty}\left(\lambda_{j, \infty}\right)^{k} a_{j, \infty}=0$. By Lemma 5.11 , we have for all $k, \lim _{j \rightarrow \infty} j^{k} a_{j, \infty}=0$. So $W_{R, \infty}(t) f$ is a classical 
solution of the wave equation, when $R$ is big enough, $W_{R, \infty}(t) f=W_{\infty}(t) f$ is independent of $R$. Since $M_{\infty}$ is a smooth manifold,

$$
e^{-t \Delta} f(x)=\frac{1}{\sqrt{4 \pi t}} \int_{-\infty}^{\infty} e^{-s^{2} / 4 t} W_{\infty}(s) f(x) d s
$$

In view of (5.57), combined with the fact $W_{\infty}$ does not increase $L^{2}$ norm and $H_{i}(x, y, t)$ converges uniformly to $H_{\infty}(x, y, t)$, we have

$$
e^{-t \Delta} f(x)=\int_{M_{\infty}} H_{\infty}(x, y, t) f(y) d y
$$

That concludes the proof.

\section{Laplacian on metric cones.}

In this section, we assume $M_{i}^{n} \stackrel{d_{G H}}{\longrightarrow} C(X)$ where $C(X)$ is a metric cone; $\operatorname{Ric}_{M_{i}^{n}} \geq 0, M_{i}^{n}$ is complete noncompact and satisfies (0.4) uniformly, $n \geq 3$.

Write $p_{\infty}$ for the pole of $C(X)$, define $r(x)=d\left(x, p_{\infty}\right)$.

Theorem 6.1. If $M_{\infty}=C(X)$, then $H_{\infty}$ is the integral kernel of the semigroup $e^{-t \Delta}$.

Proof. In view of (5.23), (5.25) and the Young's inequality, one can define a semigroup $E(t)$ on $L^{2}\left(M_{\infty}\right)$ by

$$
E(t) f(x)=\int_{M_{\infty}} H_{\infty}(x, y, t) f(y) d y .
$$

We want to compare $E(t)$ with $e^{-t \Delta}$. First, by Theorem $3.21,(1.18)$ and (5.23), one easily get

$$
G_{\infty}(x, y)=\int_{0}^{\infty} H_{\infty}(x, y, t) d t
$$

Pick any $L^{2}$ function $f$ with compact support. Write

$$
F(x)=\int_{M_{\infty}} G_{\infty}(x, y) f(y) d y .
$$


We compute

$$
\begin{aligned}
\frac{E(t) F-F}{t}= & \int_{M_{\infty}}\left(\frac{H_{\infty}(x, y, t)}{t}\right) \int_{M_{\infty}} \int_{0}^{\infty} H_{\infty}(y, z, s) f(z) d s d z d y \\
& -\frac{1}{t} \int_{M_{\infty}} \int_{0}^{\infty} H_{\infty}(x, z, s) f(z) d s d z \\
= & -\frac{1}{t} \int_{0}^{t} \int_{M_{\infty}} H_{\infty}(x, z, s) f(z) d z d s .
\end{aligned}
$$

So by (0.4), (5.23), (5.30) and the Young's inequality we have

$$
\lim _{t \rightarrow 0} \frac{E(t) F-F}{t} \rightarrow-f
$$

in $L^{2}$ and $L^{1}$.

Now we use the assumption that $M_{\infty}=C(X)$ is a noncollapsed cone. Recall the results in Section 4, we can construction a function $\phi=\phi(r)$ such that $\phi$ is a smooth function of $r$, where $r(x)=d\left(p_{\infty}, x\right)$ is the distance from the pole, and

$$
\phi(r)=1 \text { if } r<R, \phi(r)=0 \text { if } r \geq R+2, \nabla \phi \leq C_{0} \sqrt{\phi} .
$$

So on $M_{\infty}=C(X)$ we have $\Delta \phi=-\phi^{\prime \prime}-(n-1) \phi^{\prime} / r$. This function can serve as a cut off function.

We prove, if $F, f=\Delta F \in L^{2}$ have compact support, then

$$
F=\int_{C(X)} G_{\infty}(x, y) f(y) d y
$$

In fact, assume $\left\{f_{k}\right\}$ is a sequence of Lipschitz functions, $f_{k} \rightarrow f$ in $L^{2}$, and all $f_{k}$ together with $f, F$ are supported in the ball $B_{K}\left(p_{\infty}\right)$. So the function

$$
F_{k}=\int_{C(X)} G_{\infty}(x, y) f_{k}(y) d y
$$

satisfies $\Delta F_{k}=f_{k}$ by the discussion in Section 3. Consider the equation $\Delta\left(F_{k}-F\right)=f_{k}-f$, i.e.

$$
\int_{C(X)}\left\langle d F_{k}-d F, d u\right\rangle-\int_{C(X)}\left(f_{k}-f\right) u=0,
$$

for any $u \in \stackrel{\circ}{H}_{1,2}$. We set $u=\phi\left(F_{k}-F\right)$, so $d u=d \phi\left(F_{k}-F\right)+\phi\left(d F_{k}-d F\right)$. By the Schwartz inequality,

$$
\begin{aligned}
\left\|\sqrt{\phi} d\left(F_{k}-F\right)\right\|_{L^{2}}^{2} & -C_{0}\left\|\left.\left(F_{k}-F\right)\right|_{\mathrm{A}(R, R+2)}\right\|_{L^{2}}\left\|\left.\sqrt{\phi} d\left(F_{k}-F\right)\right|_{\mathrm{A}(R, R+2)}\right\|_{L^{2}} \\
& -\left\|\left.\left(f_{k}-f\right)\right|_{B_{K}}\right\|_{L^{2}}\left\|\left.\left(F_{k}-F\right)\right|_{B_{K}}\right\|_{L^{2}} \leq 0
\end{aligned}
$$


here $\mathrm{A}(R, R+2)$ is the annulus $\{x \mid R \leq r(x) \leq R+2\}$. Note we have a definite bound for $\left\|\left.F_{k}\right|_{B_{K}}\right\|_{L^{2}}$ by (1.19) and the Young's inequality. Note also by (1.19) we get, for $R>K$,

$$
\begin{aligned}
\left\|\left.\left(F_{k}-F\right)\right|_{\mathrm{A}(R, R+2)}\right\|_{L^{2}} & =\left\|\left.F_{k}\right|_{\mathrm{A}(R, R+2)}\right\|_{L^{2}}<C\left(n,\|f\|_{L^{1}}\right)\left(R^{4-2 n} R^{n-1}\right)^{1 / 2} \\
& =C\left(n,\|f\|_{L^{1}}\right) R^{(3-n) / 2}<C\left(n,\|f\|_{L^{1}}\right),
\end{aligned}
$$

since $n \geq 3$. So first, we get that $\left\|d\left(F_{k}-F\right)\right\|_{L^{2}}<\infty$ by letting $R \rightarrow \infty$. Then by letting $k \rightarrow \infty$, we have $\left\|d\left(F_{k}-F\right)\right\|_{L^{2}} \rightarrow 0$, since we can choose $R$ in (6.11) such that $\left\|\left.\sqrt{\phi} d\left(F_{k}-F\right)\right|_{\mathrm{A}(R, R+2)}\right\|_{L^{2}}$ small.

Now by the (2,2)-Poincare inequality, (0.4), (1.19) and Young's inequality, $F_{k} \rightarrow F$ in $L^{2}$ on compact sets. Also notice, on any compact sets, the right hand side of (6.9) converges to the right hand side of $(6.8)$ in $L^{2}$, by the Young inequality (however, in view of (1.19), these convergences might not be globally $L^{2}$ ). That's enough to imply (6.8).

Next we compute, for $f=\Delta F, F, f \in L^{2}$,

$$
\begin{aligned}
\| \Delta(\phi F) & -f\left\|_{L^{2}} \leq\right\| F \Delta \phi\left\|_{L^{2}}+\right\|(\phi-1) f\left\|_{L^{2}}+2\right\|<d \phi, d F>\|_{L^{2}} \\
& \leq C(n)\left\|\left.F\right|_{A(R, R+2)}\right\|_{L^{2}}+\left\|\left.f\right|_{A(R, R+2)}\right\|_{L^{2}}+C_{0}\left\|\left.d F\right|_{A(R, R+2)}\right\|_{L^{2}} .
\end{aligned}
$$

Similar to (6.11), one shows $\|d F\|_{L^{2}}<\infty$. So if $R \rightarrow \infty$, we have $\phi F \rightarrow F$ and $\Delta(\phi F) \rightarrow f=\Delta F$ in $L^{2}$. Moreover, by (6.8),

$$
\phi F(x)=\int_{C(X)} G_{\infty}(x, y) \Delta(\phi F)(y) d y .
$$

So the computation (6.5) is valid for the functions $\phi F$ and $\Delta(\phi F)$ :

$$
\lim _{t \rightarrow 0} \frac{E(t) \phi F-\phi F}{t}=-\Delta(\phi F)
$$

in $L^{2}$. We already know $E(t)$ in (6.2) is a semigroup, its infinitesimal generator is a closed operator (see [Ta]). So by the above computations, this infinitesimal generator must be the self-adjoint operator $-\Delta$ on $C(X)$.

By the discussion in the beginning of Section 4, we have an eigenfunction expansion of Laplacian on the unit cross section $X$. We denote by $\phi_{j}(j=$ $0,1,2, \ldots)$ the renormalized eigenfunctions with eigenvalues $\mu_{j}>0$, note $\phi_{0}=\operatorname{Vol}(X)^{-1 / 2} . \mu_{j} \rightarrow \infty$ when $j \rightarrow \infty$.

Put $d=\operatorname{Diam} X$. Using an argument of Gromov (see [Gr], and Theorem 4.8 of [Ch3]), we have a more precise estimate of $\mu_{j}$ :

$$
\mu_{j}>C(\tau, \kappa)^{-1} d^{-2} j^{\frac{2}{\kappa}} .
$$


On the other hand, on each ball $B_{r}\left(x_{k}\right)$ of radius $r=d / 2(j+2)$ on $X$, we define a Lipschitz function $\psi_{k}$ supported in $B_{r}\left(x_{k}\right)$ using MacShane's lemma ([Ch3], [ChCo3]):

$$
\psi_{k}\left(x_{k}\right)=r, \psi_{k}\left(\partial B_{r}\left(x_{k}\right)\right)=0, \operatorname{Lip} \psi_{k}=1,
$$

so we can follow the argument of Cheng (see p.105 of [SY]), and get

$$
\mu_{j} \leq C(\kappa) j^{2} d^{-2} .
$$

Now we can use Moser iteration, $\left|\phi_{j}\right|$ is bounded by a definite power of $j:$

$$
\left|\phi_{j}\right| \leq C(d, \kappa, \tau) j^{N(\tau, \kappa)} .
$$

Moreover, $\phi_{j}$ is Hölder continuous, see [GT], [Lin].

Write $\nu_{j}=\sqrt{\mu_{j}+\alpha^{2}}$, here $m=n-1, \alpha=(1-m) / 2$. We write $x, y$ in polar coordinates, $x=\left(r_{1}, x_{1}\right), y=\left(r_{2}, x_{2}\right)$.

\section{Theorem 6.20.}

$$
H_{\infty}=\left(r_{1} r_{2}\right)^{\alpha} \sum_{j=0}^{\infty}\left(\frac{1}{2 t}\right) e^{-\left(r_{1}^{2}+r_{2}^{2}\right) / 4 t} I_{\nu_{j}}\left(\frac{r_{1} r_{2}}{2 t}\right) \phi_{j}\left(x_{1}\right) \otimes \phi_{j}\left(x_{2}\right) .
$$

Here $I_{\nu_{j}}$ are the modified Bessel functions:

$$
I_{\nu}(z)=\left(\frac{z}{2}\right)^{\nu} \sum_{k=0}^{\infty} \frac{1}{k ! \Gamma(\nu+k+1)}\left(\frac{z}{2}\right)^{2 k} .
$$

In our case $\Delta$ is a self-adjoint operator on the whole cone $C(X)$, namely, including the pole $p_{\infty}$. By Corollary 4.25 , the separation of variable formula (4.16) works for $u=f(r) g(x)$ on the whole $C(X)$ if $u$ and $\Delta u$ are bounded on $C(X) \backslash\left\{p_{\infty}\right\}$. So the heat kernel on $M_{\infty}$ has the expression as on the right hand side of (6.21); the proof goes exactly like the classical case, see [Ch1], [Ch2] page 592, [ChTa1] and [Ta] chapter 8, we omit the details. By Theorem 6.1, we have (6.21).

By Stirling's formula, (6.16) and (6.18), we see the series (6.21) converges uniformly, when $t$ is bounded away from 0 and $r_{1}, r_{2}$ stay bounded. In particular, $H_{\infty}$ is continuous, so by Theorem 5.54 we have $H_{i} \rightarrow H_{\infty}$ uniformly. 
If one of the two points $x$ and $y$, say, $y$, is the pole $p_{\infty}$, then there is only one term in (6.21). Note $\nu_{0}=-\alpha=(m-1) / 2, m=n-1$,

$$
H_{\infty}\left(p_{\infty}, x, t\right)=\left(\frac{1}{4 \pi t}\right)^{\frac{n}{2}} e^{-\left(r^{2}\right) / 4 t} \frac{2 \pi^{n / 2}}{\Gamma(n / 2)}(\operatorname{Vol}(X))^{-1} .
$$

As a corollary, we get a new proof of Li's asymptotic formula for heat kernels [Li1]:

Corollary $6.24(\mathbf{L i})$. Assume $M^{n}$ is a complete noncompact manifold satisfying (0.4), $\operatorname{Ric}_{M^{n}} \geq 0$. Then

$$
\lim _{t \rightarrow \infty} \operatorname{Vol}\left(B_{\sqrt{t}}(p)\right) H(p, y, t)=(4 \pi)^{-n / 2} \omega_{n} .
$$

$\omega_{n}$ is the volume of the unit ball in $\mathbf{R}^{n}$.

Proof. Notice,

$$
\lim _{t \rightarrow \infty} \operatorname{Vol}\left(B_{\sqrt{t}}(p)\right) t^{-n / 2}=v_{0}=n^{-1} \operatorname{Vol}(X) .
$$

So we need to show,

$$
\lim _{t \rightarrow \infty} t^{n / 2} \operatorname{Vol}(X) H(p, y, t)=(4 \pi)^{-n / 2} n \omega_{n} .
$$

Assume $t_{i} \rightarrow \infty, M_{i}^{n}=\left(M^{n}, p, t_{i}^{-1} d x^{2}\right) \stackrel{d_{G H}}{\longrightarrow} C(X)$ for some metric cone $C(X)$; see [ChCo1]. The heat kernel $H_{i}(p, x, t)$ on $M_{i}^{n}$ is

$$
H_{i}(p, y, 1)=t^{n / 2} H(p, y, t) \text {. }
$$

Here we identify $p, x \in M_{i}^{n}$ with $p, x \in M$, however, $d_{M_{i}^{n}}(p, x)=$ $t_{i}^{-1 / 2} d_{M}(p, x), d_{M_{i}^{n}}$ is the distance on $M_{i}^{n}$. In particular, $d_{M_{i}^{n}}(p, x) \rightarrow 0$ as $i \rightarrow \infty$. Since $M_{i}^{n} \stackrel{d_{G H}}{\longrightarrow} C(X)$, by Theorem 5.54 and (6.23) we have

$$
\begin{aligned}
\lim _{t \rightarrow \infty} t^{n / 2} \operatorname{Vol}(X) H(p, y, t) & =\operatorname{Vol}(X) \lim _{i \rightarrow \infty} H_{i}(p, x, 1) \\
& =\operatorname{Vol}(X) H_{\infty}\left(p_{\infty}, p_{\infty}, 1\right)=(4 \pi)^{-n / 2} \frac{2 \pi^{n / 2}}{\Gamma(n / 2)} .
\end{aligned}
$$

We just need to recall $n \omega_{n}=2 \pi^{n / 2}(\Gamma(n / 2))^{-1}$ (see [Ta] Chapter 3).

Finally in view of the almost rigidity theorem [ChCo1], we see the above results holds for all sequences $t_{i} \rightarrow \infty$. This suffices to complete the proof.

Similarly, we get the asymptotic formula for heat kernels in [LiTW]: 
Corollary 6.30 (Li-Tam-Wang). Assume $M^{n}$ is a complete noncompact manifold satisfying (0.4), $\operatorname{Ric}_{M^{n}} \geq 0$. Then for $p \in M^{n}$, and any $R, T>0$, (6.31)

$$
\lim _{d(p, x) \rightarrow \infty} \operatorname{Vol}\left(B_{R^{-1} d(p, x)}(p)\right) H\left(p, x, T d(p, x)^{2} R^{-2}\right)=\frac{\omega_{n}}{(4 \pi T)^{n / 2} e^{R^{2} / 4 T}} .
$$

Proof. We use the same argument as in Corollary 6.24. For $x_{i}$ with $d\left(p, x_{i}\right) \rightarrow \infty$, we study the heat kernels on the sequence $M_{i}^{n}=$ $\left(M^{n}, p, R^{2} d\left(p, x_{i}\right)^{-2} d x^{2}\right)$.

We can similarly get a local asymptotic formula for $H_{\infty}$.

\section{Eigenvalues on compact limit spaces.}

We assume $M_{i}^{n} \stackrel{d_{G H}}{\longrightarrow} M_{\infty}$, with $\operatorname{Ric}_{M_{i}^{n}} \geq-(n-1) \Lambda, M_{\infty}$ compact. A point $x \in M_{\infty}$ is said to be regular, $x \in \mathcal{R}_{k}$, if all tangent cones at $x$ equal to $\mathbf{R}^{k}$; see [ChCo2].

Lemma 7.1. If $x \in \mathcal{R}_{n} \subset M_{\infty}$, then

$$
\lim _{t \rightarrow 0} H_{\infty}(x, x, t) t^{\frac{n}{2}}=(4 \pi)^{-\frac{n}{2}} .
$$

Proof. Use a similar argument as the one in Corollary 6.24.

Theorem 7.3. Assume $M_{i}^{n} \stackrel{d_{G H}}{\longrightarrow} M_{\infty}$, $\operatorname{Ric}_{M_{i}^{n}} \geq-(n-1) \Lambda$, and for some $v_{0}>0, \operatorname{Vol}\left(M_{i}^{n}\right) \geq v_{0}$. Then

$$
\lim _{j \rightarrow \infty} j^{-\frac{2}{n}} \lambda_{j, \infty}=4 \pi \Gamma\left(\frac{n}{2}+1\right)^{\frac{2}{n}} \mu_{\infty}\left(M_{\infty}\right)^{-\frac{2}{n}}
$$

Proof. In this case we don't need to renormalize the volume on $M_{i}^{n}$ (see [ChCo2]). Note for some $D$ we have $\operatorname{Diam} M_{i}^{n} \leq D, i=1,2, \ldots, \infty$, by the Bishop-Gromov inequality and (1.16), we get

$$
t^{\frac{n}{2}} H_{\infty}(x, x, t) \leq C\left(n, \Lambda, D, v_{0}\right) .
$$


Moreover, almost every point of $M_{\infty}$ is in $\mathcal{R}_{n}$. Now by Corollary 7.1, for $x \in$ $\mathcal{R}_{n}, t^{\frac{n}{2}} H_{\infty}(x, x, t) \rightarrow(4 \pi)^{-n / 2}$ when $t \rightarrow 0$. By the dominated convergence theorem,

$$
\lim _{t \rightarrow 0} t^{\frac{n}{2}} \int_{M_{\infty}} H_{\infty}(x, x, t) d x=(4 \pi)^{-\frac{n}{2}} \mu_{\infty}\left(M_{\infty}\right)
$$

Finally, by applying the Karamata Tauberian theorem (see [Ta] Chapter 8), we have

$$
\lim _{\lambda \rightarrow \infty} \lambda^{-\frac{n}{2}} N(\lambda)=\mu_{\infty}\left(M_{\infty}\right) \Gamma\left(\frac{n}{2}+1\right)^{-1}(4 \pi)^{-\frac{n}{2}}
$$

where $N(\lambda)$ is the number of eigenvalues smaller than $\lambda$. Clearly this implies the Weyl asymptotic formula (7.4).

When the limit space $M_{\infty}$ is collapsed, at present our results are less satisfactory. Recall the notion of Minkowski dimensions; see [Ma]. Assume $Z$ is a metric space. For $d>0$, let $N(Z, \epsilon) \in \mathbf{Z}$ be the minimal integer such that $Z$ can be covered by $N(Z, \epsilon)$ many balls of radius $\epsilon$. Put

$$
\begin{aligned}
& v_{d}^{-}(Z)=\liminf _{\epsilon \rightarrow 0} \epsilon^{d} N(Z, \epsilon), \\
& v_{d}^{+}(Z)=\underset{\epsilon \rightarrow 0}{\limsup \epsilon^{d} N(Z, \epsilon) .}
\end{aligned}
$$

Here $v_{d}^{ \pm}\left(M_{\infty}\right)$ can be $\infty$. The upper (lower) Minkowski dimension is defined by

$$
\overline{\operatorname{dim}}_{M i n k}(Z)\left(\underline{\operatorname{dim}}_{M i n k}(Z)\right)=\inf \left\{d \mid v_{d}^{+}(Z)=0\left(v_{d}^{-}(Z)=0\right)\right\}
$$

Lemma 7.11. There exist $E_{1}(n), E_{2}(n)>0$ such that for any $d>0$,

$$
\limsup _{t \rightarrow 0} t^{\frac{d}{2}} \int_{M_{\infty}} H_{\infty}(x, x, t) d x \leq E_{2} v_{d}^{+}\left(M_{\infty}\right),
$$

and if, in addition, $\operatorname{Ric}_{M_{i}^{n}} \geq 0$, then

$$
E_{1} v_{d}^{-}\left(M_{\infty}\right) \leq \liminf _{t \rightarrow 0} t^{\frac{d}{2}} \int_{M_{\infty}} H_{\infty}(x, x, t) d x
$$


Proof. Let $\cup_{1 \leq j \leq N\left(M_{\infty}, \sqrt{t}\right)} B_{\sqrt{t}}\left(x_{j}\right)$ be a covering of $M_{\infty}$ by a minimal set of balls of radius $\sqrt{t}$. We add up the integrals of $H_{\infty}$ on these ball an use Corollary 2.7 to get the estimates (7.12), (7.13).

Lemma 7.14. If $v_{d}^{+}\left(M_{\infty}\right)<c<\infty$, then there exist $C$ such that

$$
\lambda_{j, \infty}>C j^{\frac{2}{d}} .
$$

Proof. We can follow an argument of Gromov (see [Gr] or Theorem 4.8 in [Ch3]). Here we use the assumption $v_{d}^{+}\left(M_{\infty}\right)<c<\infty$ to estimate the number of balls that is needed to cover $M^{\infty}$.

Lemma 7.16. If $v_{d}^{-}\left(M_{\infty}\right)>c>0$, then there exist $C$ depending on $n, c$, such that

$$
\lambda_{j, \infty} \leq C j^{\frac{2}{d}} .
$$

If $k$ is the maximal integer such that $\mathcal{R}_{k} \subset M_{\infty}$ is not empty, then

$$
\lambda_{j, \infty}<C\left(M_{\infty}\right)(j)^{\frac{2}{k}}
$$

Proof. For $r>0, M_{i}^{n}$ contains $j=C(n, c) r^{-d}$ many disjoint balls of radius $r$ for $i$ big enough. The result follows by a well known argument of Cheng [Cheng]; see page 105 of [SY].

If $k$ is the maximal integer such that $\mathcal{R}_{k} \subset M_{\infty}$ is not empty, then the $k$ Hausdorff measure of $M_{\infty}$ is positive (see [ChCo3] or [Ch3]). So $v_{k}^{-}(X)>0$. By (7.17) we get (7.18).

If one can also prove for any $d>k$,

$$
\lim _{t \rightarrow 0} t^{\frac{d}{2}} \sum_{j=0}^{\infty} e^{-\lambda_{j, \infty} t}=\lim _{t \rightarrow 0} t^{\frac{d}{2}} \int_{M_{\infty}} H_{\infty}(x, x, t) d x=0,
$$

then by Lemma 7.11, $d_{M}\left(M_{\infty}\right)$, the Minkowski dimension of $M_{\infty}$ is no more than $k$. Combine with the results in [ChCo3] and [Ch3], $d_{M}\left(M_{\infty}\right)=k$. However, at present we don't know how to get (7.19). One related question is, 
Question. Is there an $\epsilon(n)>0$, such that for any $M^{n}$ with $\operatorname{Ric}_{M^{n}} \geq 0$, any eigenfunction $\phi$ of $\Delta$ and any set $E$ with $\operatorname{Vol}(E)<\epsilon \operatorname{Vol}(M)$, we have

$$
\int_{M^{n}-E} \phi^{2}>\frac{1}{2} \int_{M^{n}} \phi^{2} ?
$$

\section{References.}

[Bu] P. Buser, A note on the isoperimetric constant, Ann. Scient. Ec. Norm. Sup., 15 (1982), 213-230.

[Ch1] J. Cheeger, On the spectral geometry of spaces with cone-like singularities. Proc. Nat. Acad. Sci. USA, 76(5) (1979), 2103-2106.

[Ch2] J. Cheeger, Spectral geometry of singular Riemannian spaces, J. Differential Geom., 18 (1983), 575-657.

[Ch3] J. Cheeger, Differentiability of Lipschitz Functions on Metric Measure Spaces, GAFA, 9 (1999) 428-517.

[ChCo1] J. Cheeger and T. Colding, Lower bounds on the Ricci curvature and the almost rigidity of warped products, Ann. of Math., 144 (1996) 189-237.

[ChCo2] J. Cheeger and T. Colding, On the structure of spaces with Ricci curvature bounded below 1, J. Differential Geom., 46(3) (1997), 406-480.

[ChCo3] J. Cheeger and T. Colding, On the structure of spaces with Ricci curvature bounded below 2, preprint.

[ChCo4] J. Cheeger and T. Colding, On the structure of spaces with Ricci curvature bounded below 3, preprint.

[ChGr] J. Cheeger and D. Gromoll, The splitting theorem for manifolds of nonnegative Ricci curvature, J. Differential Geom., 6 (1971), 119128.

[CGT] J. Cheeger, M. Gromov, and M. Taylor, Finite propagation speed, kernel estimates for functions of the Laplace operator, and the geometry of complete Riemannian manifolds, J. Differential Geom., 17(1) (1982), 15-53. 
[ChTa1] J. Cheeger and M. Taylor, On the diffraction of waves by conical singularities I, Comm. Pure Appl. Math., 35(3) (1982), 275-331.

[ChY] J. Cheeger and S.-T. Yau, A lower bound for the heat kernel, Comm. Pure Appl. Math., 34(4) (1981), 465-480.

[Cheng] S.Y. Cheng, Eigenvalue comparison theorems and its geometric applications, Math. Z., 143(3) (1975), 289-297.

[CY1] S.Y. Cheng and S.-T. Yau, Differential equations on Riemannian manifolds and their geometric applications, Comm. Pure Appl. Math., 28(3) (1975), 333-354.

[Co] T. Colding, Ricci curvature and volume convergence, Ann. of Math. (2), 145(3) (1997), 477-501.

[CoMi1] T. Colding and W. Minicozzi II, Large scale behavior of kernels of Schrödinger operators, Amer. J. Math., 119(6) (1997), 1355-1398.

[CoMi2] T. Colding and W. Minicozzi II, Harmonic functions with polynomial growth, J.Differential Geom., 46(1) (1997), 1-77.

[CoMi3] T. Colding and W. Minicozzi II, Harmonic functions on manifolds, Ann. of Math. (2), 146(3) (1997), 725-747.

[Fu] K. Fukaya, Collapsing of Riemannian manifolds and eigenvalues of Laplace operator, Invent. Math., 87(3) (1987), 517-547.

[Gr] M. Gromov, Metric structures for Riemannian and NonRiemannian Spaces, Progress in Mathematics, 152, Birkhäuser Boston, Inc., Boston, MA, 1999.

[GT] D. Gilbarg and N. Trudinger, Elliptic Partial Differential Equations of Second Order, Springer-Verlag, New York, 1983.

[HaKo] P. Hajlasz and P. Koskela, Sobolev meets Poincare, C.R. Acad. Sci. Paris Ser. I Math., 320(10) (1995), 1211-1215.

[Hir] M. Hirsch, Differential Topology Springer-Verlag, New York, 1976.

[J] D. Jerison, The Poincare inequality for vector fields satisfying Hörmander's condition, Duke Math. J., 53 (1985), 309-338. 
[KK1] A. Kasue and H. Kumura, Spectral convergence of Riemannian manifolds, Töhoku Math. J. (2), 46(2) (1994), 147-179.

[KK2] A. Kasue and H. Kumura, Spectral convergence of Riemannian manifolds, II, Töhoku Math. J. (2), 48(1) (1996), 71-120.

[Li1] P. Li, Large time behavior of the heat equation on complete manifolds with non-negative Ricci curvature, Ann. Math., 124 (1986), 1-21.

[Li2] P. Li, Lecture notes on geometric analysis, Lecture Notes Series, 6, Seoul National University, Seoul, 1993.

[LiT1] P. Li and L.F. Tam, Symmetric Green's function on complete manifolds, Amer. J. Math., 109 (1987), 1129-1154.

[LiT2] P. Li and L.-F. Tam, Complete surfaces with finite total curvature, J. Differential Geom., 33 (1991), 139-168.

[LiTW] P. Li, L.-F. Tam, and J. Wang, Sharp bounds for the Green's function and the heat kernel, Math. Res. Lett., 4(4) (1997), 589-602.

[LiY1] P. Li and S.-T. Yau, Estimates of eigenvalues of a compact Riemannian manifold, in 'Geometry of the Laplace operator,' 205-239, Proc. Sympos. Pure Math., XXXVI, AMS, Providence, RI, 1980.

[LiY2] P. Li and S.-T. Yau, On the parabolic kernel of the Schrödinger operator, Acta Math., 156 (1986), 153-201.

[Lin] F. Lin, Elliptic Partial Differential Equations, Courant Lecture Notes in Mathematics, 1 (1997).

[Ma] P. Mattila, Geometry of sets and measures in Euclidean spaces, Cambridge University Press, Cambridge, 1995.

[Per] G. Perelman, A complete Riemannian manifold of positive Ricci curvature with Euclidean volume growth and nonunique asymptotic cone, Comparison geometry, 165-166, Cambridge Univ. Press, Cambridge, 1997.

[SY] R. Schoen and S.-T. Yau, Lectures on Differential Geometry, International Press, Cambridge, MA, 1994. 
[Ta] M. Taylor, Partial Differential Equations, Volume 1, 2, 3, SpringerVerlag, New York, NY, 1996.

Courant Institute of Mathematical Sciences

251 Mercer Street, New York, NY 10012

E-mail address: dingyu@cims.nyu.edu

Received June 7, 2000. 